\title{
Frequency-Domain Design of Overcomplete Rational-Dilation Wavelet Transforms
}

\author{
İlker Bayram, Student Member, IEEE and Ivan W. Selesnick, Senior Member, IEEE
}

\begin{abstract}
The dyadic wavelet transform is an effective tool for processing piecewise smooth signals; however, its poor frequency resolution (its low Q-factor) limits its effectiveness for processing oscillatory signals like speech, EEG, and vibration measurements, etc. This paper develops a more flexible family of wavelet transforms for which the frequency resolution can be varied. The new wavelet transform can attain higher Q-factors (desirable for processing oscillatory signals) or the same low Q-factor of the dyadic wavelet transform. The new wavelet transform is modestly overcomplete and based on rational dilations. Like the dyadic wavelet transform, it is an easily invertible 'constantQ' discrete transform implemented using iterated filter banks and can likewise be associated with a wavelet frame for $L_{2}(\mathbb{R})$. The wavelet can be made to resemble a Gabor function and can hence have good concentration in the time-frequency plane. The construction of the new wavelet transform depends on the judicious use of both the transform's redundancy and the flexibility allowed by frequency-domain filter design.
\end{abstract}

\section{INTRODUCTION}

The dyadic wavelet transform (WT) is an easily-invertible 'constant-Q' transform that is very effective for the sparse representation of piecewise smooth signals, like a scan-line from a typical photographic image [11]. This property has made the dyadic WT a popular tool for several signal and image processing applications (coding, denoising, deblurring, sharpening, etc). However, the dyadic WT is less effective for processing signals of more oscillatory nature, like speech, EEG, and physical vibration measurements, etc. Such signals are quasi-periodic over short-time intervals; and when analyzing/filtering such signals one generally needs better frequency resolution than that provided by the dyadic WT. Indeed, the dyadic WT has a very low Q-factor ${ }^{1}$ and poor frequency resolution, as illustrated in Fig. 1. Other transforms, like the short-time Fourier transform, cosine modulated filter banks, and wavelet packets, are often used for oscillatory-type signals instead of the dyadic WT. But these transforms are not constant-Q. This paper develops a family of wavelet (constantQ) transforms attaining a range of Q-factors; in particular, compared to the dyadic WT, the Q-factor can be made higher and the frequency resolution can be made finer. (The proposed WT can also attain the same low Q-factor as the dyadic WT.)

Copyright (c) 2009 IEEE. Personal use of this material is permitted. However, permission to use this material for any other purposes must be obtained from the IEEE by sending a request to pubs-permissions @ieee.org.

I. Bayram and I. W. Selesnick are with the Department of Electrical and Computer Engineering, Polytechnic Institute of New York University, Brooklyn, NY 11201

Phone: (718) 260-3416, Fax: (718) 260-3906,

E-mail: ibayra01@students.poly.edu, selesi@poly.edu.

${ }^{1}$ The Q-factor of a band-pass filter is the ratio of its center frequency to its bandwidth.



(a)

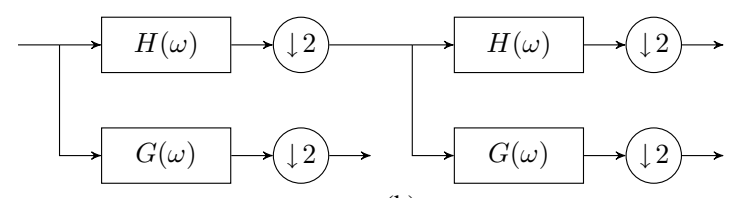

(b)

Fig. 1. The critically-sampled dyadic discrete wavelet transform has a low Q-factor. (a) Frequency decomposition and (b) iterated filter bank implementation.

For many signal processing applications, overcomplete wavelet transforms outperform critically-sampled wavelet transforms. Overcomplete WTs (or 'frames') expand an $N$ point signal to a set of $M$-coefficients with $M>N$ [22]. Several overcomplete invertible WTs are available, like the undecimated WT (UWT), the dual-tree complex WT $(\mathbb{C W T})$, the double-density WT, and others [29], [19], [30], [14]. However, many of these WTs ${ }^{2}$ attain over-completeness by increasing only the temporal sampling in some or all frequency bands. But, in order to better utilize the redundancy of an overcomplete WT it can be beneficial to also reduce the frequency spacing between adjacent frequency bands. Additionally, most of the existing overcomplete WTs achieve either a limited range of redundancy factors $(M / N)$ or can be much more overcomplete than is necessary or practical. The family of wavelet transforms developed in this paper attain over-completeness by increasing sampling in both time and frequency. Moreover, the proposed family of WTs provides a rich range of redundancy factors.

Based on the engineering literature, most current discrete wavelet transform implementations are based on FIR filters. Indeed, much of the development of wavelet-based signal processing algorithms grew from Daubechies' construction of FIR-based orthonormal wavelet bases [16]. However, the FFT-

\footnotetext{
${ }^{2}$ We exclude the continuous 'integral' wavelet transform because we restrict our interest to fully-discrete transforms that are easily and stably inverted.
} 
based implementation of filter banks and WTs offers greater design flexibility; and this flexibility has been exploited in the development of certain WTs so as to attain attributes not possible with FIR filters [34], [18], [24]. Likewise, the family of WTs introduced in this paper is based on a frequencydomain design (the filters do not have rational transfer functions) and our implementation is based on the FFT. We note that although the filters are not FIR, the time-domain filter response decays rapidly and the wavelets are well localized in time and frequency.

The family of overcomplete wavelet transforms introduced in this paper is based on rational (non-dyadic) dilations. This WT is developed for discrete-time data, is approximately shiftinvariant, and is easily invertible (in fact, self-inverting in the sense of [31] - the frame is a 'tight' frame). By using a dilation factor close to one, one obtains a WT where the wavelet is gradually dilated from scale to scale. In addition, the introduced WT is flexible - a range of Q-factors and redundancy factors can be attained.

Most previous research on wavelet transforms with rational dilations consider only the critically-sampled case [6], [23], [12], [5], [7]. An exception is our previous work [3] in which we developed overcomplete rational-dilation filter banks and WTs with FIR filters. However, the family of rational-dilation WTs proposed here, designed in the frequency-domain and implemented using the FFT, have several advantages over the WTs developed in [3]. First, in [3] each frequency band (or 'scale') is represented by a number of signals produced by different band-pass filters; the signals need to be interlaced together to form the subband signal - an inconvenience. Second, the construction in [3] involves polynomial matrix spectral factorization, which while being straightforward in principle, is more involved than the frequency-domain approach proposed here. Consequently, in [3] we were unable to produce WTs with good frequency resolution (high Qfactor), even though that was one of our motivations. Third, the approach proposed in this paper has greater flexibility; in particular, the redundancy factor of the transform is not completely determined by the dilation factor as it is in [3].

The close relationship, between invertible wavelet transforms for discrete data and multiresolution anaylsis (MRA) on the real line, is well recognized and appreciated. For the dyadic case, self-inverting FIR FBs are related to compactlysupported tight wavelet frames on the real line. Unfortunately for rational-dilation WTs, no MRA with rational dilation factors can be constructed based on self-inverting FIR FBs [6]; that is, there is no unique well defined wavelet on the real line associated with discrete rational-dilation wavelet transforms implemented using FIR filters. However, in this paper we will show that a tight rational-dilation wavelet frame on the real line can be constructed using the proposed bandlimited filters ${ }^{3}$ using a single wavelet. This is an extension of the construction by Auscher [1] of an orthonormal MRA with $q-p$ wavelets.

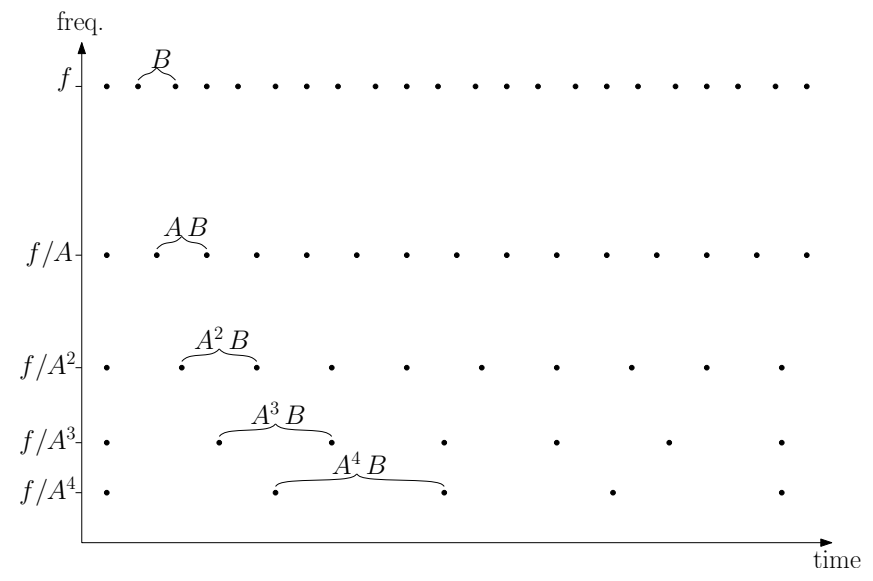

Fig. 2. The time-frequency sampling lattice of a discrete wavelet transform implemented via an iterated two-channel filter bank, as in Figs. 1 and 3. The parameters $A$ and $B$ are determined by the sampling factor of the low-pass branch and the redundancy of the filter bank respectively.

\section{A. Sampling the Time-Frequency Plane}

The manner in which the analysis/synthesis functions cover the time-frequency (T-F) plane, as illustrated in Fig. 2, provides a useful way to understand the relationships among various wavelet transforms. For example, the dyadic WT, realized using the filter bank illustrated in Fig. 1b, can be understood to sample the T-F plane with $\{A=2, B=2\}$ in Fig. 2. In contrast, the critically-sampled rational-dilation WT, realized using the iterated FB in Fig. 3a, sets $\{A=q / p, B=$ $q /(q-p)\}$

Overcomplete wavelet transforms sample the T-F plane more densely. For example, the double-density WT [29] doubles the temporal density of the dyadic WT and maintains its frequency spacing, leading to $\{A=2, B=1\}$ in Fig. 2. In this paper, we propose a family of WTs realized using the iterated FB in Fig. 3b, which sets $\{A=q / p, B=s\}$.

Our previous work on overcomplete rational-dilation wavelet transforms [3] sets $\{A=q / p, B=1\}$ and is therefore less flexible than the WT proposed here $(s=1$ in [3]). In particular, the proposed WT can sample the T-F plane more coarsely than the WT of [3] and it can therefore be less redundant. Indeed, the proposed WT is redundant by $p /(s(q-p))$, whereas the WT of [3] is redundant by $p /(q-p)$. The latter redundancy can be large even for modest rational sampling factors. For example, with $p=7, q=8$, which is relevant for classification of audio signals [12], the WT of [3] is redundant by a factor of 7 . Such a high redundancy might be higher than is desired or necessary. Using $s=3$ reduces the redundancy to $7 / 3$.

In addition to affecting the redundancy, $s$, in the filter bank of Fig. 3b, also affects the Q-factor and the time-bandwidth product. We will demonstrate that, for a given rational dilation factor, $q / p$, there is a trade-off between the Q-factor and the time-bandwidth product.

\footnotetext{
${ }^{3}$ For the filter bank in Fig. 4, we will show that perfect reconstruction (PR) cannot be achieved using filters with rational transfer functions.
} 


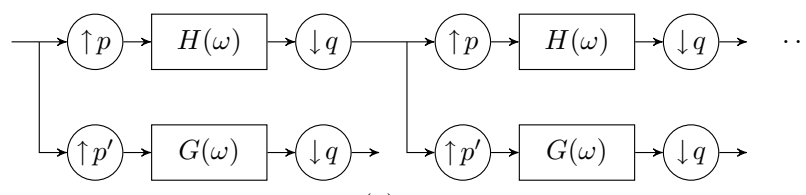

(a)

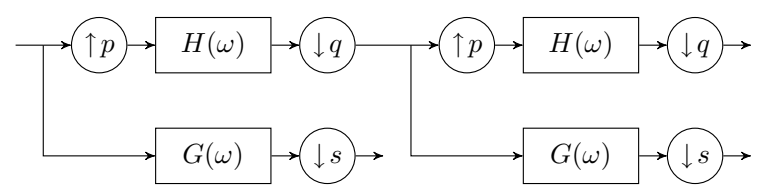

(b)

Fig. 3. Rational-dilation discrete wavelet transforms. (a) The criticallysampled rational WT (with $p^{\prime}=q-p$ ), (b) The overcomplete rational WT investigated in this paper. The dilation factor is $q / p$.

\section{B. Prior Constant-Q Transforms}

Numerous methods have been proposed to obtain constantQ transforms with high Q-factors (see [8] and the references therein). For example, Oppenheim et al. describe a method that maps a discrete sequence to another whose discrete-time Fourier transform (DTFT) is related to the former via a frequency warping, thereby allowing one to obtain an unequally spaced frequency sampling [28]. In this direction, Smith and Abel [32] use a bilinear map as the warping function to approximate the Bark frequency warping. Makur and Mitra discuss in [25] the warped DFT which samples the unit circle on points that are not equally spaced as required by the conventional DFT. In this regard, the constant-Q transform introduced by Brown in [8] may be interpreted (even though Brown's work precedes [25]) as a short-time Fourier transform that uses a sliding window with the warped DFT instead of the conventional DFT (also see [9] for an implementation of the inverse transform).

A second approach to obtaining constant-Q transforms with variable Q-factor is to approximate the T-F lattice with simpler building blocks. For instance, Diniz et al. [17] provide an efficient implementation of a transform called the bounded-Q fast filter bank, inspired by the bounded-Q transform introduced by Mont-Reynaud [27]. The bounded-Q transform first splits the spectrum into geometrically distributed frequency bins and then splits each of these bins uniformly, achieving a piecewise linear frequency decomposition. In a similar vein, Karmakar et al. [21] propose a criterion to select optimal wavelet packet trees to approximate the Bark scale.

The rational-dilation wavelet transform proposed in this paper provides an alternative, with attractive properties, to the methods above that set out to achieve a constant-Q analysis with a high Q-factor. First, it is a tight frame, hence the inverse is given by the transpose of the forward transform and can be implemented as efficiently, a property lacked by the first group of transforms. Second, it is a true constant-Q transform, in contrast to the methods in the second group. Therefore, we believe that the introduced rational-dilation WT may be a useful addition to the inventory of transforms designed for the task of constant-Q analysis.

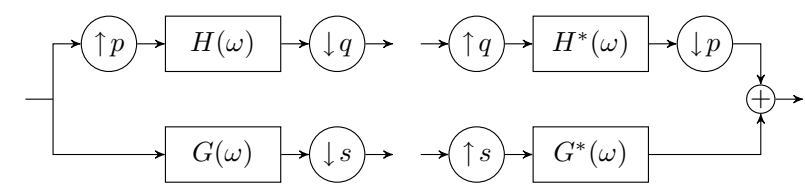

Fig. 4. Analysis and synthesis filter banks for the implementation of the proposed rational-dilation wavelet transform. The dilation factor is $q / p$, a rational number.

\section{Notation and Conventions}

We denote discrete-time sequences by lower case letters as in $a(n)$ with $n \in \mathbb{Z}$. The discrete-time Fourier transform (DTFT) of $a(n)$ is denoted by $A(\omega)$ and is given by $A(\omega)=$ $\sum_{n} a(n) \exp (-j \omega n)$. Note that $A(\omega)$ is $2 \pi$ periodic in $\omega$. Unless otherwise stated, we assume all discrete-time signals are real valued, so that specifying a DTFT on $\omega \in[0, \pi]$ will determine the DTFT on $\omega \in[-\pi, \pi]$. Therefore, we restrict our attention to $\omega \in[0, \pi]$ so as to simplify some of the notation. The Fourier transform of a function $f(t)$, with $t \in \mathbb{R}$, is given by $\hat{f}(\omega)=\int_{-\infty}^{\infty} f(t) e^{-j \omega t} d t$.

\section{Organization}

In Section II, we derive the perfect reconstruction conditions and propose a specific set of perfect reconstruction filters. In Sections III and IV, we show that for the proposed filters, the rate changer and related iterated filter banks can be written so as to simplify the analysis of their behavior. In Section V we provide examples. In Section VI we examine the Q-factor and time-bandwidth product of the proposed WT. In Section VII, we present another interpretation of the rational rate changer and discuss the underlying wavelet frame for $L_{2}(\mathbb{R})$.

\section{Perfect Reconstruction Conditions}

In this section we derive the perfect reconstruction conditions for the filter bank in Fig. 4. This FB is the basic element of the proposed wavelet transform. The parameters $p, q$, and $s$ are positive integers satisfying $1 \leq p<q$ and $p / q+1 / s \geq 1$. We also assume $p$ and $q$ are coprime. We will show that PR cannot be attained using filters with rational transfer functions. Hence our filter bank implementation will be based on the FFT. For this reason, we formulate the PR conditions in the frequency domain.

Let us consider the system in Fig. 5. We have,

$$
U(\omega)=X(a \omega)
$$

$$
\begin{aligned}
& V(\omega)=\frac{1}{b}\left[F(\omega) U(\omega)+F\left(\omega+\frac{2 \pi}{b}\right) U\left(\omega+\frac{2 \pi}{b}\right)+\cdots\right. \\
& \left.+F\left(\omega+(b-1) \frac{2 \pi}{b}\right) U\left(\omega+(b-1) \frac{2 \pi}{b}\right)\right] F^{*}(\omega),
\end{aligned}
$$

and

$$
\begin{aligned}
Y(\omega)=\frac{1}{a}\left[V\left(\frac{\omega}{a}\right)+V\left(\frac{\omega}{a}\right.\right. & \left.+\frac{2 \pi}{a}\right)+\cdots \\
& \left.+V\left(\frac{\omega}{a}+(a-1) \frac{2 \pi}{a}\right)\right] .
\end{aligned}
$$


Noting that

$$
U\left(\frac{\omega}{a}+k \frac{2 \pi}{b}+n \frac{2 \pi}{a}\right)=X\left(\omega+a k \frac{2 \pi}{b}\right),
$$

we obtain

$$
Y(\omega)=\sum_{k=0}^{b-1} C_{k}(\omega) X\left(\omega+a k \frac{2 \pi}{b}\right)
$$

where

$$
\begin{aligned}
C_{k}(\omega) & =\frac{1}{a b}\left[F\left(\frac{\omega}{a}+k \frac{2 \pi}{b}\right) F^{*}\left(\frac{\omega}{a}\right)\right. \\
+ & F\left(\frac{\omega}{a}+k \frac{2 \pi}{b}+\frac{2 \pi}{a}\right) F^{*}\left(\frac{\omega}{a}+\frac{2 \pi}{a}\right)+\cdots \\
+F & \left.\left(\frac{\omega}{a}+k \frac{2 \pi}{b}+(a-1) \frac{2 \pi}{a}\right) F^{*}\left(\frac{\omega}{a}+(a-1) \frac{2 \pi}{a}\right)\right] .
\end{aligned}
$$

We can now write the output of the FB in Fig. 4 as,

$Y(\omega)=\sum_{k=0}^{q-1} L_{k}(\omega) X\left(\omega+p k \frac{2 \pi}{q}\right)+\sum_{k=0}^{s-1} M_{k}(\omega) X\left(\omega+k \frac{2 \pi}{s}\right)$

where

$$
\begin{aligned}
L_{k}(\omega) & =\frac{1}{p q} \sum_{n=0}^{p-1} H\left(\frac{\omega}{p}+k \frac{2 \pi}{q}+n \frac{2 \pi}{p}\right) H^{*}\left(\frac{\omega}{p}+n \frac{2 \pi}{p}\right), \\
M_{k}(\omega) & =\frac{1}{s}\left[G\left(\omega+k \frac{2 \pi}{s}\right) G^{*}(\omega)\right] .
\end{aligned}
$$

Proposition 1: For the FB in Fig. 4, there exist filters with non-zero rational transfer functions achieving PR if and only if $p+1=q=s$.

Proof: See Appendix A.

Notice that $p+1=q=s$ implies the FB is orthonormal. Therefore, in order to obtain perfect reconstruction filters for the overcomplete case, we must use filters which do not have rational transfer functions.

We remark that if,

$$
\begin{aligned}
& L_{k}(\omega)=0 \quad \text { for } k=1,2, \ldots, q-1, \\
& M_{k}(\omega)=0 \quad \text { for } k=1,2, \ldots, s-1, \\
& L_{0}(\omega)+M_{0}(\omega)=1
\end{aligned}
$$

then PR is granted ${ }^{4}$.

\section{A. Proposed perfect reconstruction filters}

In this section we propose a low-pass filter $H(\omega)$ and a high-pass filter $G(\omega)$ satisfying the perfect reconstruction conditions derived above. The two filters will have ideal passbands and ideal stop-bands; however, the filters will not be ideal 'brick-wall' filters because of the presence of transition bands. In fact, the transition bands are important because a 'brick-wall' filter will have a sinc-type time-domain response with poor decay, which we wish to avoid.

\footnotetext{
${ }^{4}$ It can be shown that these conditons are also necessary if $q$ and $s$ are coprime.
}

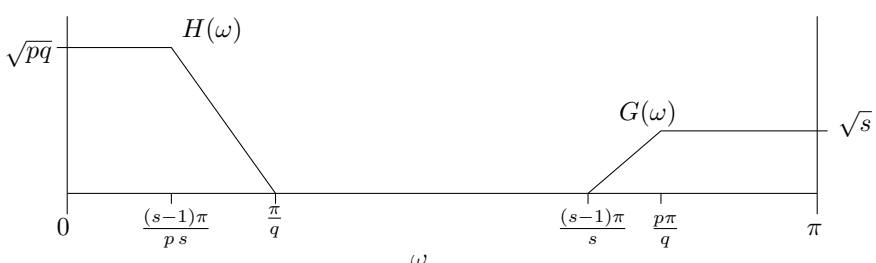

(a)

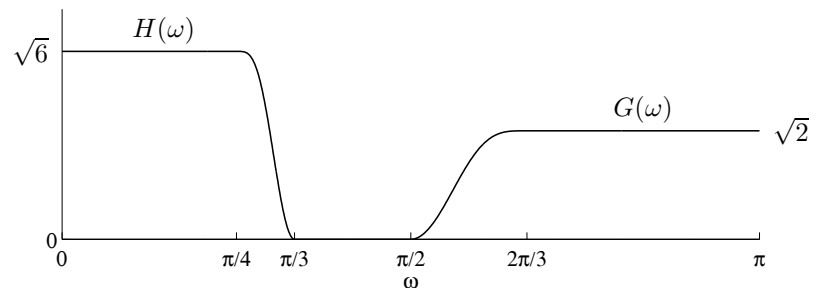

(b) $p=2, q=3, s=2$.

Fig. 6. (a) Properties of proposed perfect reconstruction filters for the filter bank in Fig. 4. (b) Proposed filters with dilation factor $q / p=3 / 2$ and $s=2$.

If we let,

$$
H(\omega)=0, \quad \text { for } \omega \in\left[\frac{\pi}{q}, \pi\right]
$$

then it can be shown that,

$$
\begin{aligned}
& L_{k}(\omega)=0, \quad k=1,2, \ldots, q-1 \\
& L_{0}(\omega)=\frac{1}{p q}\left|H\left(\frac{\omega}{p}\right)\right|^{2} \quad \text { for } \omega \in[0, \pi] .
\end{aligned}
$$

Similarly, if

$$
G(\omega)=0, \quad \omega \in\left[0,\left(1-\frac{1}{s}\right) \pi\right]
$$

then,

$$
\begin{aligned}
& M_{k}(\omega)=0, \quad k=1,2, \ldots, s-1 \\
& M_{0}(\omega)=\frac{1}{s}|G(\omega)|^{2} \quad \text { for } \omega \in[0, \pi] .
\end{aligned}
$$

In other words, with the choices (13) and (16), the only condition for PR is, by (12),

$$
\frac{1}{p q}\left|H\left(\frac{\omega}{p}\right)\right|^{2}+\frac{1}{s}|G(\omega)|^{2}=1 \quad \text { for } \omega \in[0, \pi] .
$$

Equations (13) and (19) imply that

$$
|G(\omega)|=\sqrt{s} \quad \text { for } \omega \in\left[\frac{p}{q} \pi, \pi\right] .
$$

Similarly, (16) and (19) imply

$$
|H(\omega)|=\sqrt{p q} \quad \text { for } \omega \in\left[0,\left(1-\frac{1}{s}\right) \frac{\pi}{p}\right] .
$$

Notice that (13) and (21) determine $|H(\omega)|$ on all $\omega$ except $[(1-1 / s) \pi / p, \pi / q]$. Likewise, (16) and (20) determine $G(\omega)$ on all $\omega$ except $[(1-1 / s) \pi, p \pi / q]$. These will be the transition bands of $H(\omega)$ and $G(\omega)$. From (19), the transition band of $|G(\omega)|$ is completely determined by the transition band of $|H(\omega)|$. These constraints on the filters are depicted in Fig. 6a for reference. 


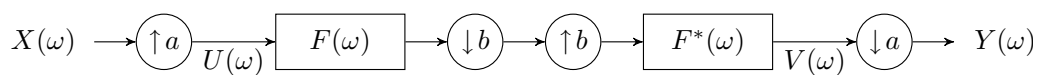

Fig. 5. One channel of the rational filter bank in Fig. 4.

It is easy to check that, provided

$$
\frac{p}{q}+\frac{1}{s}>1,
$$

the transition bandwidths in Fig. 6 are positive. That is, provided the FB is overcomplete, $H(\omega)$ and $G(\omega)$ really do have transition bands. The width of the transition band will be important because it will influence the decay of $h(n)$ and $g(n)$ and of the analysis/synthesis functions of the designed wavelet transform.

Even though Fig. 6 defines transition bands for the filters $H(\omega)$ and $G(\omega)$, with different widths, we remark that for the low-pass branch, the filter $H(\omega)$ acts not on the input signal but on the up-sampled input signal. In Section IV, we rewrite the low-pass branch so as to clarify the filtering operation.

\section{B. Transition Bands}

The transition band of $H(\omega)$ should be carefully specified so that both $h(n)$ and $g(n)$ are reasonably well time-localized, especially because the proposed frequency responses $H(\omega)$ and $G(\omega)$ have ideal stop-bands and pass-bands. On its transition band, $[(1-1 / s) \pi / p, \pi / q]$, the frequency response $H(\omega)$ can be set arbitrarily, as long as $|H(\omega)| \leq \sqrt{p q}$. We define the transition function $\theta(\omega)$ on $\omega \in[0, \pi]$ with $|\theta(\omega)| \leq 1$, and set

$$
H(\omega)=\sqrt{p q} \theta\left(\frac{\omega-a}{b}\right) \text { for } \omega \in\left[\left(1-\frac{1}{s}\right) \frac{\pi}{p}, \frac{\pi}{q}\right],
$$

where $a$ and $b$ are such that $(\omega-a) / b$ maps $[0, \pi]$ to the transition band of $H(\omega)$. Specifically,

$$
\begin{aligned}
& a=\left(1-\frac{1}{s}\right) \frac{\pi}{p}, \\
& b=\frac{1}{q}-\left(1-\frac{1}{s}\right) \frac{1}{p} .
\end{aligned}
$$

We also define the complementary transition function $\theta_{c}(\omega)$ as

$$
\theta_{c}(\omega):=\sqrt{1-\theta^{2}(\omega)}
$$

Then, the proposed PR filters can be written as

$$
\begin{aligned}
& H(\omega)= \begin{cases}\sqrt{p q} & \omega \in\left[0,\left(1-\frac{1}{s}\right) \frac{\pi}{p}\right), \\
\sqrt{p q} \theta\left(\frac{\omega-a}{b}\right) & \omega \in\left[\left(1-\frac{1}{s}\right) \frac{\pi}{p}, \frac{\pi}{q}\right), \\
0 & \omega \in\left[\frac{\pi}{q}, \pi\right],\end{cases} \\
& G(\omega)= \begin{cases}0 & \omega \in\left[0,\left(1-\frac{1}{s}\right) \pi\right), \\
\sqrt{s} \theta_{c}\left(\frac{\omega-p a}{p b}\right) & \omega \in\left[\left(1-\frac{1}{s}\right) \pi, \frac{p}{q} \pi\right), \\
\sqrt{s} & \omega \in\left[\frac{p}{q} \pi, \pi\right] .\end{cases}
\end{aligned}
$$

Furthermore, we propose for the transition function, $\theta(\omega)$, that the Daubechies filter [16] with two vanishing moments be used (to be precise, the DTFT magnitude thereof, restricted to $[0, \pi]$ and normalized so that $\theta(\omega) \leq 1$ ). Specifically, we set

$$
\theta(\omega)=\frac{1}{2}(1+\cos (\omega)) \sqrt{2-\cos (\omega)} \text { for } \omega \in[0, \pi] .
$$

We make this choice for $\theta(\omega)$ because then the transition bands of $H(\omega)$ and $G(\omega)$ will have the same behavior, namely for $\theta(\omega)$ in (28) we have $\theta_{c}(\omega)=\theta(\pi-\omega)$. Figure 6b illustrates $H(\omega)$ and $G(\omega)$ with this transition function for $p=2, q=3$, and $s=2$.

Using for $\theta(\omega)$ a Daubechies filter having a higher number of vanishing moments will increase the differentiability of $H(\omega)$. However, if the number of vanishing moments is large, then the frequency response $H(\omega)$ will be closer to a 'brick wall' and the time-domain response $h(n)$ will be closer to a sinc function which has poor (slow) decay. Because we wish that the analysis/synthesis functions of the constructed wavelet transform have good time-frequency localization properties, we use a Daubechies filter with a small number of vanishing moments. Note that the proposed construction need not use a Daubechies filter - any set of perfect reconstruction filters could be used in the construction.

FFT filter bank implementation. Our implementation of the proposed rational-dilation filter bank for finite-length input signals is based on the FFT. Specifically, our implementation consists of using the FFT to implement circular convolution for low-pass and high-pass filtering. Our implementation provides perfect reconstruction for signals of any length.

For perfect reconstruction of the wavelet transform, the length of the input signal at each level should be a multiple of $q$ and of $s$; that is, the signal length should be a multiple of the least common multiple of $q$ and $s$, denoted $\operatorname{lcm}(q, s)$. This condition can be understood as follows: If a discretetime periodic signal with period $N$ is down-sampled by $q$, the resulting signal will be periodic with period $N / q$ only if $N$ is divisible by $q$. Because the two-channel analysis filter bank contains down-samplers by both $q$ and $s$, the input signal should be divisible by both $q$ and $s$. Otherwise, circular convolution filtering does not readily lead to the perfect reconstruction property.

In case the signal length is not a multiple of $\operatorname{lcm}(q, s)$, we zero-pad the signal to the next multiple of $\operatorname{lcm}(q, s)$. We perform the necessary zero-padding at each level of the wavelet transform. As a consequence of the zero-padding, each subband signal may be a few samples longer than the minimum; however, the transform is already overcomplete so the additional few samples caused by zero-padding at each level will usually be negligible. As a second consequence of the zero-padding, the length of the reconstructed signal may be a few samples longer than the original signal but these additional signal values will be equal to zero in the absence of wavelet-domain processing.

Instead of zero-padding at each stage of the wavelet transform we could zero-pad the original signal. However, zeropadding the original signal instead of level-by-level would require zero-padding to the next multiple of $\operatorname{lcm}\left(q^{J}, s\right)$ where $J$ is the number of levels. This can lead to much more zero-padding than level-by-level zero-padding. Avoiding that 


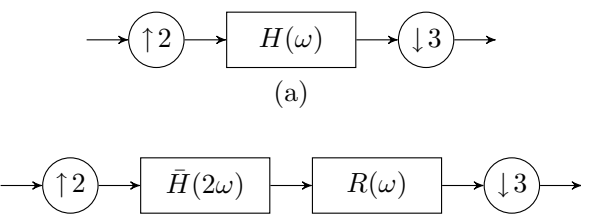

(b)

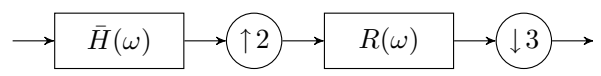

(c)

Fig. 7. Provided that $H(\omega)$ is bandlimited to $\pi / 3$, and $\bar{H}(\omega)$ and $R(\omega)$ are defined as in (30) and (31), the three systems are equivalent.

length extension is especially important for the high Q-factor case because a high Q-factor transform will generally have more levels (large $J$ ) and $q$ will be greater in comparison to a low Q-factor transform - leading to excessive zeropadding. Our implementation avoids this. On the other had, our implementation can aggravate the artificial discontinuity introduced by periodic boundary extensions. We assume the signal is sufficiently longer than the wavelet at each level so that periodic boundary artifacts are minor.

Although the described FFT-implementation may be computationally suboptimal for some lengths, it does provide perfect reconstruction without requiring the signal be of a special length. We have used this implementation for speech and EEG signals longer than 10,000 samples.

\section{REWRITING THE FILTER BANK}

In order to make more explicit the filtering operation the low-pass channel performs on the input signal, in this section we rewrite the filter bank in Fig. 4 so that the filtering precedes the up-sampler. In general, the order of the up-sampler and the low-pass filter $H(\omega)$ in Fig. 4 can not be exchanged. However, for the proposed low-pass filter $H(\omega)$ in Section II-A, the exchange of the up-sampler and low-pass filter is possible because the proposed low-pass filter is appropriately bandlimited.

Rewriting the filter bank in Fig. 4 so that the filter acts directly on the input signal instead of on the up-sampled input signal clarifies the behavior of the filter bank. For, when a $p$ fold up-sampling precedes the filter $H(\omega)$ as in Fig. 4, the input signal is convolved not with the impulse response $h(n)$, but with each of the down-sampled sub-sequences $h(q n+k)$ for $0 \leq k \leq q-1$. Therefore, each sub-sequence should be assessed (its frequency response, frequency-resolution, timedomain ringing, time-frequency localization, etc). The bandlimited property of the proposed low-pass filter $H(\omega)$ not only simplifies the perfect reconstruction equations, it also facilitates the assessment of the designed filter bank.

Consider the rate changer in Fig. 7a. For clarity, we set $p=2$ and $q=3$. Suppose that $H(\omega)$ is bandlimited to $\pi / 3$,

$$
H(\omega)=0 \quad \text { for } \omega \in[\pi / 3, \pi],
$$

as illustrated in Fig. 8. If we define $\bar{H}(\omega)$ as a $2 \pi$-periodic
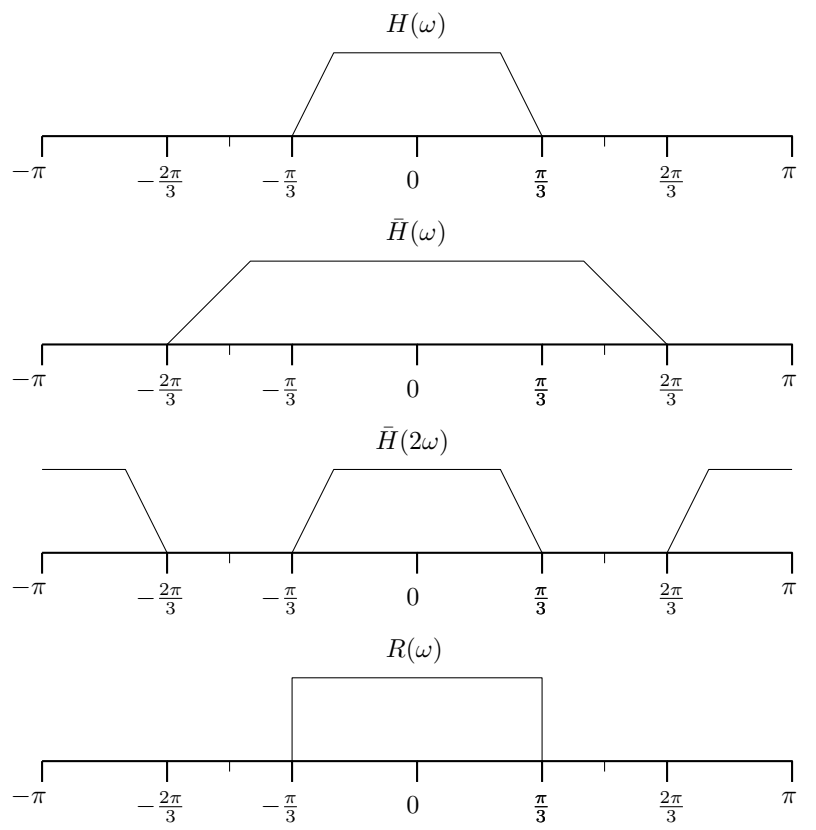

Fig. 8. The frequency response $H(\omega)$ may be written as $\bar{H}(2 \omega) R(\omega)$, if it is bandlimited to $\pi / 3$.

function,

$$
\bar{H}(\omega):=\frac{1}{2} H\left(\frac{\omega}{2}\right) \quad \text { for } \omega \in[0, \pi],
$$

and $R(\omega)$ as an ideal low-pass filter,

$$
R(\omega):= \begin{cases}2 & \omega \in[0, \pi / 3], \\ 0 & \omega \in(\pi / 3, \pi] .\end{cases}
$$

then we can write, as illustrated in Fig. 8,

$$
H(\omega)=\bar{H}(2 \omega) R(\omega),
$$

as depicted in Fig. 7b. Using noble identities, this system is equivalent to the one shown in Fig. 7c. Hence we can interpret the system in Fig. 7a as filtering followed by an ideal rate changer, ${ }^{5}$ provided $H(\omega)$ is bandlimited to $\pi / 3$.

This filter bank identity is valid for general $p$ and $q$, provided $H(\omega)$ is bandlimited to $\pi / q$. Figure 9 illustrates the general FB identity where $\bar{H}(\omega)$ and $R_{p, q}(\omega)$ are defined as

$$
\begin{aligned}
\bar{H}(\omega) & =\frac{1}{p} H\left(\frac{\omega}{p}\right) \quad \text { for } \omega \in[0, \pi], \\
R_{p, q}(\omega) & = \begin{cases}p & \omega \in[0, \pi / q], \\
0 & \omega \in(\pi / q, \pi] .\end{cases}
\end{aligned}
$$

Because the filter $H(\omega)$ proposed in Section II-B is bandlimited to $\pi / q$, we can apply the identity to the rational-dilation filter bank in Fig. 4. We obtain the FB in Fig. 10. The filter $\bar{H}(\omega)$ is bandlimited to $p \pi / q$. The system following $\bar{H}(\omega)$ is an ideal rate changer.

${ }^{5} \mathrm{By}$ an ideal rate changer we mean a $p$-fold up-sampler, followed by an ideal low-pass filter, followed by a $q$-fold down-sampler. The cut-off frequency of the low-pass filter should be $\min \{\pi / p, \pi / q\}$. See for example Eqn. 13.27 in [26]. 


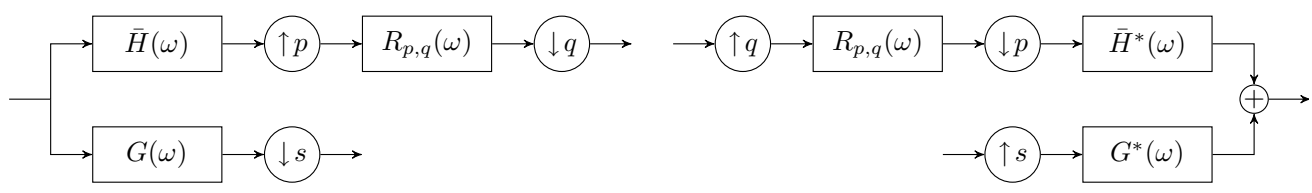

Fig. 10. An equivalent filter bank to the one in Fig. 4 provided $H(\omega)$ is bandlimited to $\pi / q$.

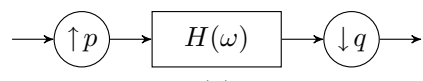

(a)

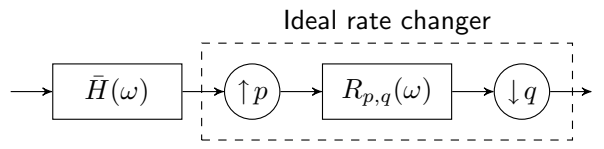

(b)

Fig. 9. If $H(\omega)$ is bandlimited to $\pi / q$, then the two systems are equivalent. The frequency responses, $\bar{H}(\omega)$ and $R_{p, q}(\omega)$, are defined in (33) and (34).

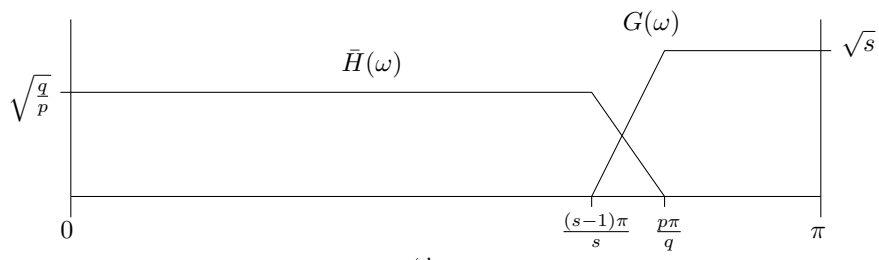

(a)

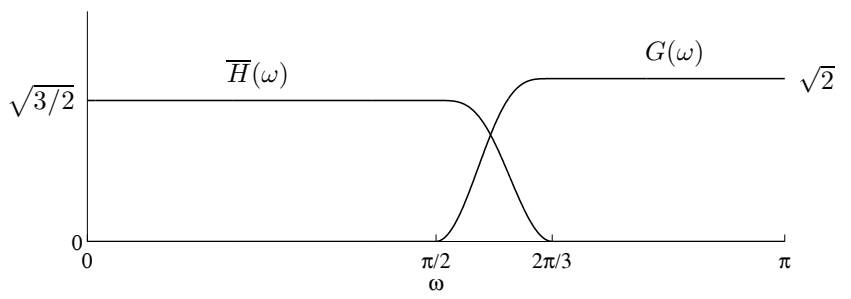

(b) $p=2, q=3, s=2$

Fig. 11. (a) Properties of the filters $\bar{H}(\omega)$ and $G(\omega)$. The transition bands are the same. (b) Proposed filters with dilation factor $3 / 2$ and $s=2$.

Rewriting the filter bank in Fig. 4 as in Fig. 10 reveals the role of the low-pass filter in the system. Notice that, because the low-pass filter $\bar{H}(\omega)$ in Fig. 10 band-limits the input signal to $p \pi / q$, the ideal rate changer causes no aliasing. This is in contrast to a critically-sampled filter bank. It is the filter $\bar{H}(\omega)$ that acts directly on the input signal. Thus it is the transition band of $\bar{H}(\omega)$ and not that of $H(\omega)$, that plays a direct part in determining the analysis/synthesis functions of the implemented DWT. In fact, if we replace $H(\omega)$ with $\bar{H}(\omega)$ in Fig. 6, then the transition bands coincide, as illustrated in Fig. 11.

Note also, that for fixed $p$ and $q$, reducing $s$ will widen the transition band, which in turn will lead to a faster decay of the time-domain response $h(n)$. The influence of $s$ will be illustrated by examples in Section V.

\section{ITERATED FILTER BANKS}

In Section III, we rewrote the filter bank in Fig. 4 as the filter bank in Fig. 10 so that the low-pass filter acts directly on the input signal instead of on an up-sampled version thereof. In this section, we will similarly rewrite the iterated filter bank. First, we consider the iterated rate changer with a generic lowpass filter. Second, we assume the filters are bandlimited like the filters proposed in Section II-B.

Consider the rate changer iterated for $j$ stages, as illustrated in Fig. 12a. It can be shown using noble identities [33] that this system is equivalent to the one in Fig. 12b where

$$
H_{j}(\omega)=\prod_{k=0}^{j-1} H\left(q^{j-1-k} p^{k} \omega\right)
$$

In using this formula to obtain $H_{j}(\omega)$, one must keep in mind that $H(\omega)$ is periodic by $2 \pi$.

To further simplify the system in Fig. 12b, we wish to rewrite it as in Fig. 7c; namely, we wish to exchange the order of the up-sampler and the low-pass filter. Provided $H(\omega)$ is bandlimited to $\pi / q$ we have such a result in the following proposition. The proposition is expected because $H(\omega)$ is bandlimited to the Nyquist rate for the rate changer.

Proposition 2: Suppose $H(\omega)$ is bandlimited to $\pi / q$. Then, $H_{j}(\omega)$ as defined in (35) is bandlimited to $\pi / q^{j}$. More precisely, we have

$$
H_{j}(\omega)= \begin{cases}\prod_{k=0}^{j-1} H\left(q^{j-1-k} p^{k} \omega\right) & \omega \in\left[0, \pi / q^{j}\right], \\ 0 & \omega \in\left(\pi / q^{j}, \pi\right] .\end{cases}
$$

Proof: See Appendix B.

We remark that (36) is not the same as (35). In general, (35) does not ensure that $H_{j}(\omega)$ is bandlimited, whereas (36) does. Defining

$$
\begin{aligned}
\bar{H}_{j}(\omega) & =\frac{1}{p^{j}} H_{j}\left(\frac{\omega}{p^{j}}\right) \text { for } \omega \in[0, \pi], \\
R_{p^{j}, q^{j}}(\omega) & = \begin{cases}p^{j} & \omega \in\left[0, \pi / q^{j}\right], \\
0 & \omega \in\left(\pi / q^{j}, \pi\right]\end{cases}
\end{aligned}
$$

and using the filter bank identity in Fig. 9, we have that all three systems in Fig. 12 are equivalent. The filter $\bar{H}_{j}(\omega)$ is bandlimited to $(p / q)^{j} \pi$.

We now consider a band-pass channel of the iterated filter bank, as illustrated in Fig. 13a. In this case, if we define

$$
G_{j+1}(\omega)=H_{j}(\omega) G\left(q^{j} \omega\right),
$$

then the two systems in Fig. 13a and 13b are equivalent (using, again, the noble identities). In addition, if $H(\omega)$ is bandlimited to $\pi / q$, then by Prop. $2, H_{j}(\omega)$ is bandlimited to $\pi / q^{j}$, and in turn, $G_{j+1}(\omega)$ is also bandlimited to $\pi / q^{j}$. Therefore,

$$
G_{j+1}(\omega)= \begin{cases}H_{j}(\omega) G\left(q^{j} \omega\right) & \omega \in\left[0, \pi / q^{j}\right] \\ 0 & \omega \in\left(\pi / q^{j}, \pi\right] .\end{cases}
$$




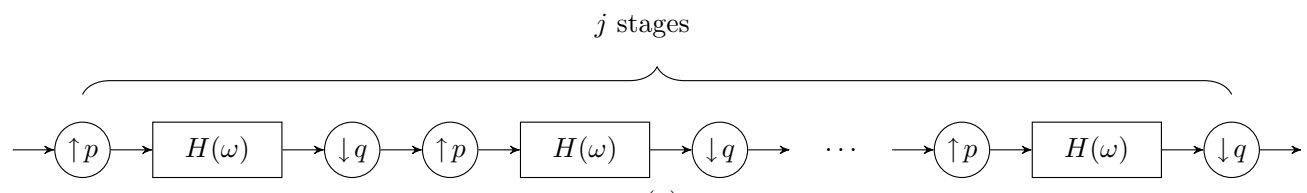

(a)

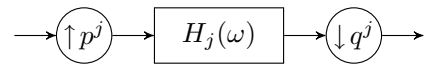

(b)

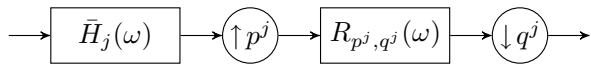

(c)

Fig. 12. The systems in (a) and (b) are equivalent. If $H(\omega)$ is bandlimited to $\pi / q$ then all three systems are equivalent.

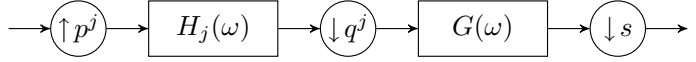

(a)

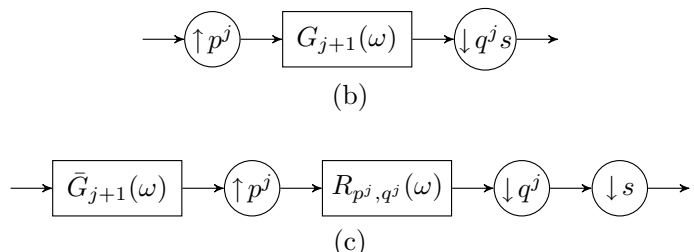

(c)

Fig. 13. The systems in (a) and (b) are equivalent. If $H(\omega)$ is bandlimited to $\pi / q$ then all three systems are equivalent.

Using the filter bank identity illustrated in Fig. 9, we define $\bar{G}_{j+1}(\omega)$ as

$$
\bar{G}_{j+1}(\omega)=\frac{1}{p^{j}} G_{j+1}\left(\frac{\omega}{p^{j}}\right) \text { for } \omega \in[0, \pi],
$$

and we rewrite the system in Fig. 13b as Fig. 13c. All three systems in Fig. 13 are equivalent. The filter $\bar{G}_{j+1}(\omega)$ in Fig. 13c acts directly on the input signal instead of an upsampled version thereof. The filtered signal is then processed with an ideal rate changer, and subsequently down-sampled by $s$.

The wavelet. For the rational-dilation wavelet transform, we define the wavelet in the frequency-domain as

$$
\hat{\psi}(\omega)=\lim _{j \rightarrow \infty}\left(\frac{p}{q}\right)^{j / 2} \bar{G}_{j}\left(\left(\frac{p}{q}\right)^{j} \omega\right) .
$$

We will show in Section VII that specific shifts and dilations of the wavelet $\psi(t)$ yield a tight frame for $L_{2}(\mathbb{R})$.

Redundancy factor. The redundancy of the overcomplete rational-dilation wavelet transform is found as follows. We first define the redundancy factor of the iterated FB with $j$ stages, $\operatorname{Red}_{j}(p, q, s)$, as the number of analysis FB coefficients per input sample, which can be shown to be

$$
\operatorname{Red}_{j}(p, q, s)=\frac{1}{s} \frac{1-(p / q)^{j+1}}{1-p / q}+\left(\frac{p}{q}\right)^{j+1} .
$$

The redundancy of the wavelet transform (iterated FB) is given by

$$
\operatorname{Red}(p, q, s)=\lim _{j \rightarrow \infty} \operatorname{Red}_{j}(p, q, s)=\frac{1}{s} \frac{1}{1-p / q}
$$

\section{EXAMPLES}

This section presents four examples to illustrate the frequency responses of the iterated filter bank, the wavelet, and the effect of the parameters $p, q$, and $s$ thereon. Each example is based on the iterated filter bank in Fig. 4 and the filters proposed in II-B. The transition function $\theta(\omega)$ is given by (28).

Example 1: In this example, we set $p=2, q=3, s=2$. The dilation factor is $3 / 2$ and the redundancy is $3 / 2$. Iterating the FB yields the frequency responses $\bar{G}_{j}(\omega)$ shown in Fig. 14 . Note that each frequency response is exactly constant over part of its pass-band (i.e. has a flat top). The wavelet $\psi(t)$ and its Fourier transform $\hat{\psi}(\omega)$ are also shown in the figure. The wavelet somewhat resembles a sinc wavelet; however, it decays more rapidly.

Example 2: We set $p=2, q=3, s=1$. The dilation factor is $3 / 2$ and the redundancy is 3. Iterating the FB yields the frequency responses shown in Fig. 15. Compared with Example 1, the frequency responses are less frequency selective. In addition, the band-pass filters are not exactly constant over any part of the pass-band (except for the highpass filter at stage 1 , which is constant for $3 \pi / 4 \leq \omega \leq \pi$ ). The wavelet $\psi(t)$ and its Fourier transform $\hat{\psi}(\omega)$ are also shown in the figure. Compared with Example 1, the wavelet has fewer oscillations and much less 'ringing'. In fact, the wavelet closely resembles the Mexican hat function (the second derivative of the Gaussian function). This example mirrors the example in [3] with 2 vanishing moments; in that example the redundancy was also 3 and the analysis/synthesis functions also resembled the Mexican hat function. (For the FIR solutions in [3] there is no uniquely defined wavelet, so we refer instead to the discrete-time analysis/synthesis functions.)

Example 3: We set $p=7, q=8, s=5$. The dilation factor is $8 / 7 \approx 1.14$ and the redundancy is $8 / 5=1.6$. The iterated frequency responses, the wavelet $\psi(t)$ and its Fourier transform $\hat{\psi}(\omega)$ are shown in Fig. 16. Compared with Examples 1 and 2, the Q-factor is higher; there are more bandpass filters covering the same frequency range. Like Example 1 , each frequency response is exactly constant over part of its pass-band. The wavelet has more oscillations than the wavelets of Examples 1 and 2; accordingly, this wavelet has a higher Q-factor. Also, note that this wavelet has some ringing; that is, there are several oscillations present outside the primary oscillatory interval.

Example 4: We set $p=7, q=8, s=3$. The dilation factor is $8 / 7 \approx 1.14$ and the redundancy is $8 / 3 \approx 2.67$. 

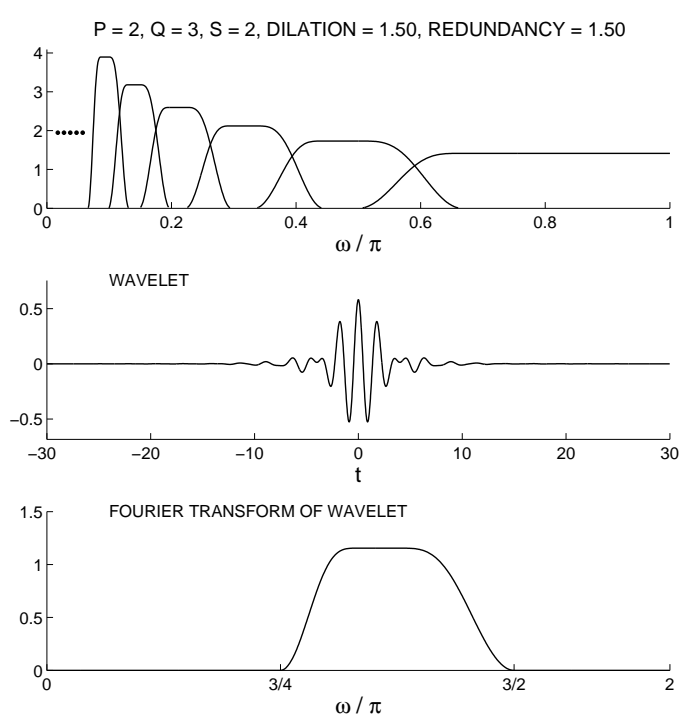

Fig. 14. Example 1: The iterated frequency responses, the wavelet $\psi(t)$, and its Fourier transform $\hat{\psi}(\omega)$.
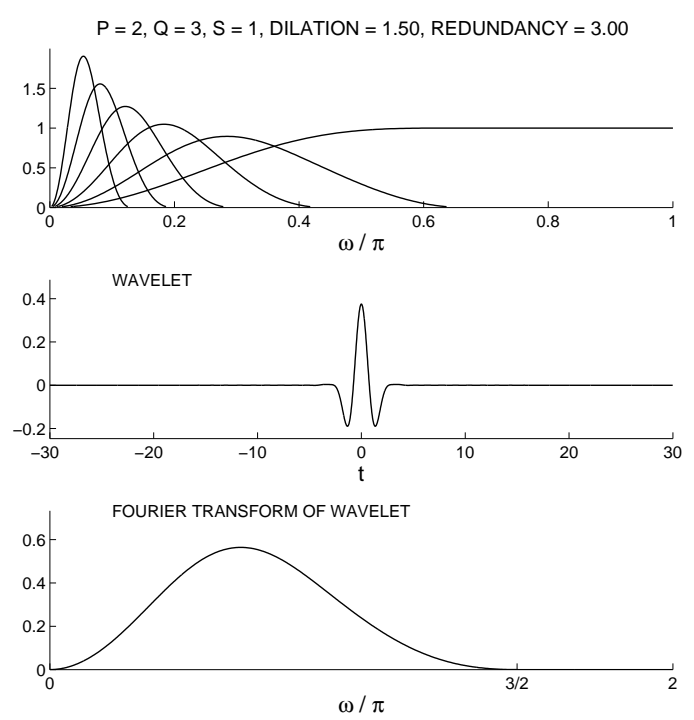

Fig. 15. Example 2: The wavelet resembles the 'Mexican hat' function.

The iterated frequency responses, the wavelet $\psi(t)$ and its Fourier transform $\hat{\psi}(\omega)$ are shown in Fig. 17. Like Example 2, the band-pass filters do not have flat tops; their shape is more Gaussian. This is because the smaller value of $s$, compared with Example 3, leads to $H(\omega)$ and $G(\omega)$ having wider transitions bands. The wavelet does not have the ringing behavior present in Example 3. The wavelet resembles a cosine function multiplied by a Gaussian function; that is, a Gabor function. The approximate Gaussian shape is present in both the time-domain and frequency-domain. Accordingly, the wavelet in this example is better localized in the timefrequency plane (the Gabor function being optimally localized) than Example 3. Compared to Examples 1 and 2, the Q-factor is higher (the wavelet has more oscillations than the wavelets of Examples 1 and 2). Note that these properties (good timefrequency localization, resemblance of the wavelet to a Gabor
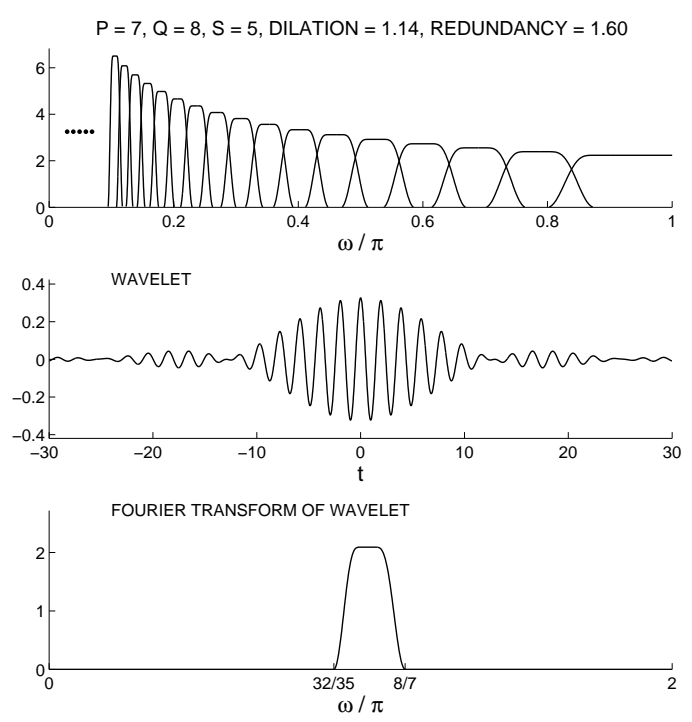

Fig. 16. Example 3: The wavelet has a higher Q-factor than Examples 1 and 2 .
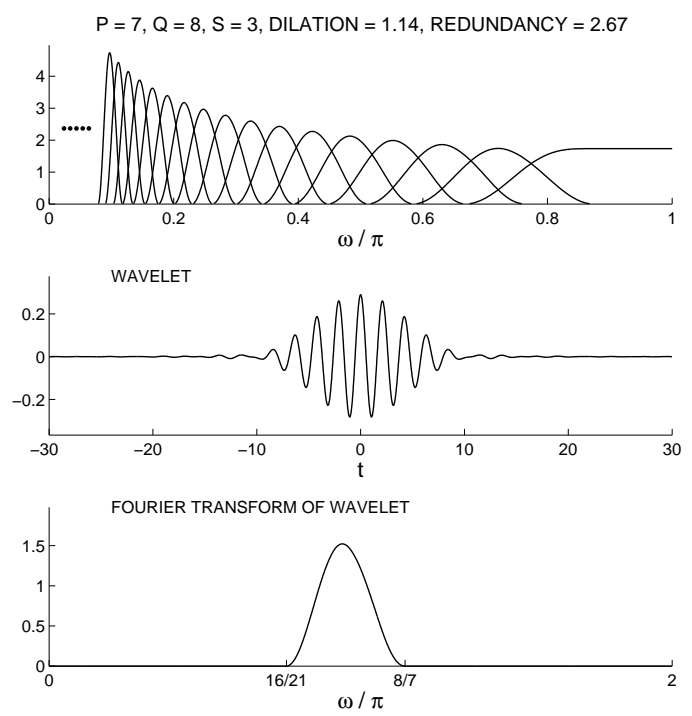

Fig. 17. Example 4: The wavelet has a higher Q-factor than Examples 1 and 2, and less 'ringing' than Example 3.

function, absence of ringing, and high Q-factor) are attained with a modest factor of redundancy (less than 3).

Note that for both dilations factors, $q / p=3 / 2$ and $q / p=8 / 7$, increasing the redundancy (decreasing $s$ ) reduced the ringing present in the wavelet and reduced the Q-factor. Finding the best parameters $p, q$, and $s$ so at so obtain (approximately) a desired Q-factor and desired absence of ringing, may require inspecting the wavelet generated by several different sets of parameter.

\section{Quality-Factor and Time-Bandwidth Product}

\section{A. The Quality-Factor}

One motivation for developing a rational-dilation wavelet transform is the ability to achieve a high Q-factor fullydiscrete self-inverting wavelet transform. Example 4 in Section $\mathrm{V}$ illustrates that for suitably chosen parameters $p$, and $s$ 
the proposed transform can achieve high Q-factors with good time-frequency localization. In this section, we examine the Q-factor as a function of the parameters.

To find the Q-factor of the proposed wavelet transform we find the Q-factor of the band-pass filters $\bar{G}_{j}(\omega)$. We will use the filters $H(\omega)$ and $G(\omega)$ proposed in Section II-B and the formulas developed in Section IV. For $H(\omega)$ and $G(\omega)$, bandlimited as in (13) and (16), it can be shown using (36), (40), and (41) that the frequency support of $\bar{G}_{j}(\omega)$ is

$$
\operatorname{supp}\left\{\bar{G}_{j}(\omega)\right\}=\left[\frac{p^{j-1}}{q^{j-1}}\left(1-\frac{1}{s}\right) \pi, \frac{p^{j-1}}{q^{j-1}} \pi\right], \quad j>1 .
$$

In addition, if

$$
1-\frac{1}{s}>\left(\frac{p}{q}\right)^{2}
$$

then $\bar{G}_{j}(\omega)$ will be exactly constant over part of its pass-band (it will have a flat top) as in Examples 1 and 3.

When (46) is satisfied (i.e. when $\bar{G}_{j}(\omega)$ has a flat top), then the two transition bands of $\bar{G}_{j}(\omega)$ are

$$
\left[\frac{p^{j-2}}{q^{j-2}}\left(1-\frac{1}{s}\right) \pi, \frac{p^{j-1}}{q^{j-1}} \pi\right] \text { and }\left[\frac{p^{j-1}}{q^{j-1}}\left(1-\frac{1}{s}\right) \pi, \frac{p^{j}}{q^{j}} \pi\right]
$$

for $j>1$. If the transition function $\theta(\omega)$ is chosen such that $|\theta(\omega)|^{2}$ is half-band (for example (28)) then the pass-band edges, defined by the half-power frequencies, are the midpoints of the transition bands (47). The resonant frequency of $\bar{G}_{j}(\omega)$, denoted $\mathrm{RF}_{j}$ and defined as the geometric mean of the left and right pass-band edges, is given by

$$
\mathrm{RF}_{j}=\frac{p^{j-2}}{q^{j-2}} \frac{1}{2}\left(1-\frac{1}{s}+\frac{p}{q}\right) \sqrt{\frac{p}{q}} \pi .
$$

Using (47), the bandwidth of $\bar{G}_{j}(\omega)$ is given by

$$
\mathrm{BW}_{j}=\frac{p^{j-2}}{q^{j-2}} \frac{1}{2}\left(1-\frac{1}{s}+\frac{p}{q}\right)\left(1-\frac{p}{q}\right) \pi .
$$

The Q-factor, given by the ratio of the resonant frequency to the bandwidth, is

$$
Q_{j}=\frac{\mathrm{RF}_{j}}{\mathrm{BW}_{j}}=\sqrt{\frac{p}{q}} \frac{1}{1-p / q}, \quad j>1 .
$$

Since $Q_{j}$ is a constant, independent of the stage index $j$, the iterated FB is a constant-Q transform (excluding the first stage).

The Q-factor formula (50) is valid only when (46) is satisfied, ie. when $\bar{G}_{j}(\omega)$ has a flat top, as in Examples 1 and 3. When the band-pass filters do not have flat tops, it is difficult to find a formula for the Q-factor and we calculate the Q-factor numerically. Note that the formulas (44) and (46) imply that $\bar{G}_{j}(\omega)$ will never have a flat top when the redundancy is greater than two. If one wishes that the wavelet be well localized in time-frequency and relatively free of ringing, then one should select the parameters $p, q$ and $s$ so that the redundancy is great than two in order to avoid the 'flat-top' behaviour.

Figure 18 illustrates the Q-factor for numerous sets $(p, q, s)$. In the figure, each set of connected points corresponds to a single $(p, q)$ pair and represents the $\mathrm{Q}$-factor as a function

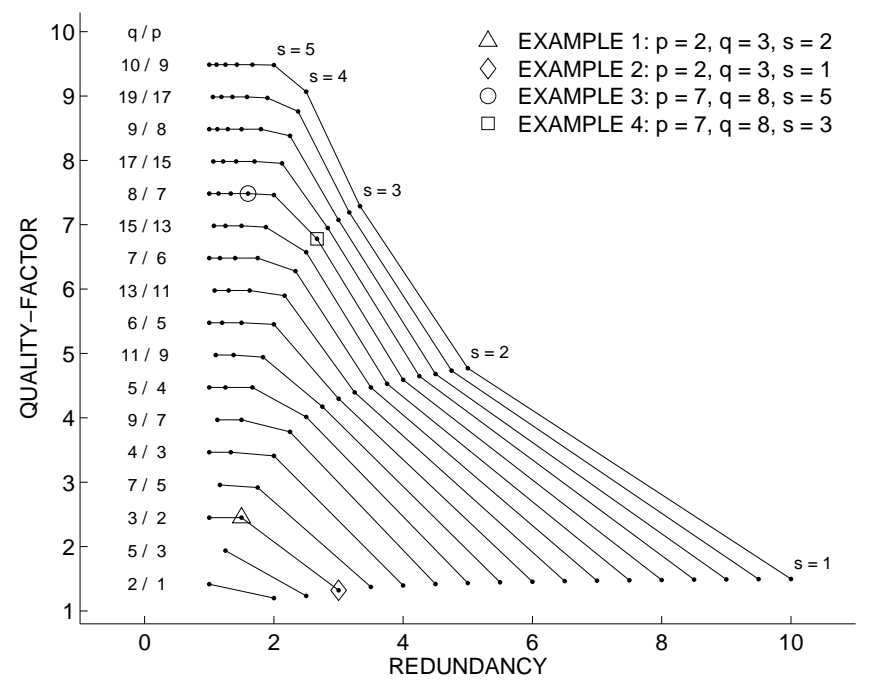

Fig. 18. The quality-factor of the proposed wavelet transform for various parameter sets $(p, q, s)$. Given a $p$ and $q$, the Q-factor decreases as the redundancy increases. Examples 1-4 are highlighted in the figure.

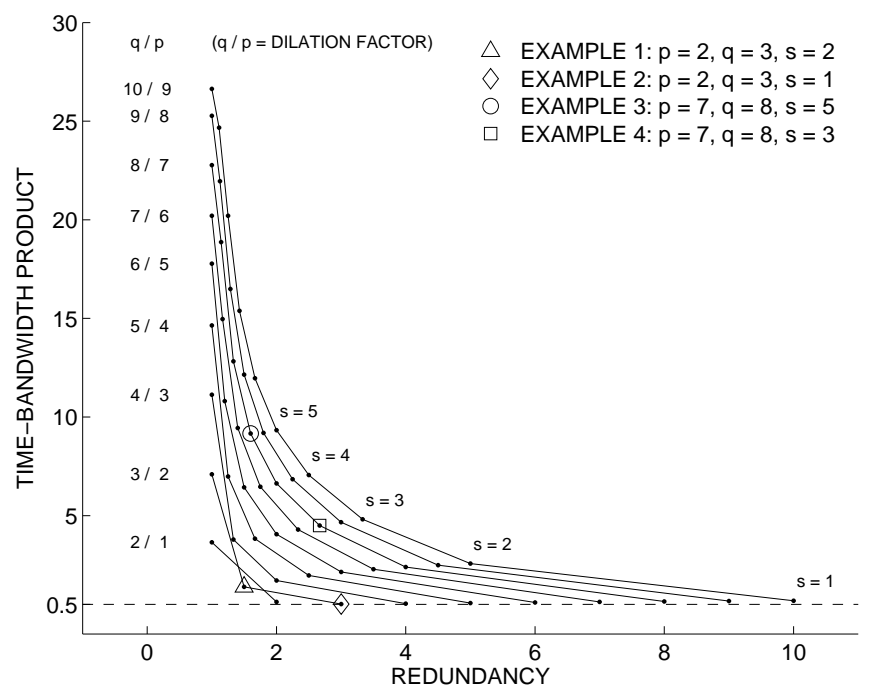

Fig. 19. The time-bandwidth product of the wavelet $\psi(t)$ for various parameter sets $(p, q, s)$. Given $p$ and $q$, the time-bandwidth product decreases as the redundancy increases. The dashed line at 0.5 is the lower bound for the time-bandwidth product. Examples 1-4 are highlighted in the figure.

of redundancy (44). The parameters $(p, q, s)$ used to produce Examples 1-4 in Section V are indicated in the figure. The figure indicates that increasing the redundancy (by reducing $s$ ) has the effect of reducing the Q-factor, a behaviour reflected in Examples 1-4.

\section{B. Time-Frequency Localization}

For the proposed wavelet transform, Section VI-A examined the frequency resolution as a function of the parameters $p, q$, and $s$, by computing the Q-factor for numerous sets $(p, q, s)$. We can also examine the wavelets' time-frequency localization as a function of the parameters by computing the time-bandwidth product [15]. Figure 19 illustrates the timebandwidth product, $\sigma_{t} \sigma_{\omega}$, for numerous sets $(p, q, s)$. The 


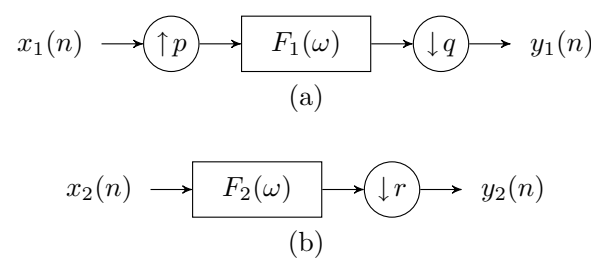

Fig. 20. The role of the filter in the first system in (a) is more implicit compared to the one in (b).

frequency variance $\sigma_{\omega}^{2}$ is computed using only the positive $\omega$ axis because the wavelet $\psi(t)$, being a real-valued band-pass function, has a two-sided spectrum. We compute the timebandwidth product numerically.

As Fig. 19 illustrates, as we increase the redundancy (decrease $s$ ) the time-bandwidth decreases and approaches its optimal value of $1 / 2$. (Note $\sigma_{t} \sigma_{\omega} \geq 1 / 2$.) The examples in Section V reflect the behavior illustrated in Fig. 19. Examples 1 and 2 have the same dilation factor of 1.5, with Example 2 having the higher redundancy (lower $s$ ). Accordingly, Example 2 has the lower Q-factor and the smaller time-bandwidth product; in fact, its time-bandwidth product is practically optimal.

\section{REINTERPRETING THE RATE CHANGER}

In this section, we provide another interpretation for the system comprised of an up-sampler, filter and a down-sampler. This is slightly more general than the interpretation in Section III and allows us to define fractional down-samplers, leading to an understanding of rational FBs parallel to integer downsampled FBs. This in turn proves useful for deriving the underlying wavelet frame for $L_{2}(\mathbb{R})$ and understanding its relation with the introduced rational DWT, similar to the relation between the integer-dilation wavelet frames and integer downsampled DWTs.

In Fig.20a, the output signal values are the inner products of the input signal values with the filter's impulse response values. Specifically, we can write,

$$
y_{1}(n)=\left\langle x_{1}(k), f_{1}(q n-p k)\right\rangle .
$$

Notice that $f_{1}(p n+q)$ for $q=1,2, \ldots, p-1$ give the polyphase components of $f_{1}(n)$. We thus see that the output of the system in Fig. 20a is obtained by sampling the convolution of the input with not the filter, but its polyphase components. In general, it is not easy to determine the relationship among the polyphase components of the filter. However, the particular choice (13) leads to a certain relation that in turn will allow us to interpret the low-pass analysis channel of the rational FB as a filtering followed by a fractional down-sampling operation, which we will describe now.

Once again, to gain insight, let us start with the simple system shown in Fig. 21a. Defining the polyphase components of $F(z)$ via,

$$
F(z)=F_{0}\left(z^{2}\right)+z^{-3} F_{1}\left(z^{2}\right),
$$

it can be shown that this system is equivalent to the one shown in Fig. 21b. Now, if we choose $F(\omega)$ such that $F(\omega)=0$

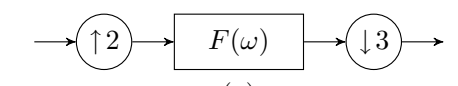

(a)

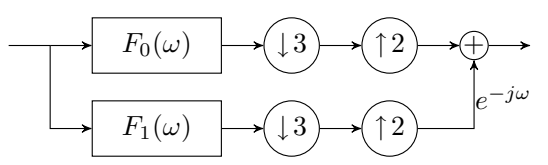

(b)

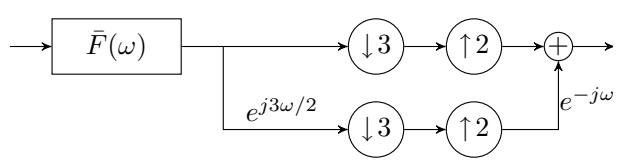

(c)

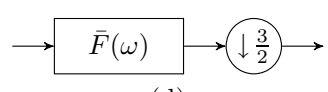

(d)

Fig. 21. The analysis channel. $F_{0}(z)$ and $F_{1}(z)$ are polyphase components of $F(z)$. Setting $F(\omega)=F(\omega / 2)$, the systems in (a), (b), (c) are equivalent. We define the fractional down-sampler by $3 / 2$ to be the system following $\bar{F}(\omega)$. This implies that all the systems are equivalent.

for $\omega \in[\pi / 2, \pi]$, and define $\bar{F}(\omega)=(1 / 2) F(\omega / 2)$ for $\omega \in$ $[0, \pi]$, then it turns out that $F_{0}(\omega)=\bar{F}(\omega)$ and $F_{1}(\omega)=$ $e^{j 3 \omega / 2} \bar{F}(\omega)$. This establishes the equivalence of Fig. 21b and 21c.

Now consider the system following $\bar{F}(\omega)$ in Fig. 21c. Suppose we apply this system to an input $x(n)$, and call the output $y(n)$. For integer $n$, the upper channel sets $y(2 n)=x(3 n)$. Interpreting $e^{j \omega \omega / 2}$ as an advance operator by $3 / 2$, we can likewise say that the lower channel sets $y(2 n+1)=x(3 n+3 / 2)$. Combining these, we get, formally, $y(n)=x(3 n / 2)$. This observation motivates us to define a fractional down-sampling operation by $3 / 2$. Using this definition, we thus say that the system in Fig. 21a is equivalent to the one in Fig. $21 \mathrm{~d}$.

For general sampling factors $p, q$, we have,

Proposition 3: For $p, q$ coprime, suppose that the $p$ polyphase components of $H(z)$, namely $H_{k}(z)$, are defined via

$$
H(z)=\sum_{k=0}^{p-1} z^{-q k} H_{k}\left(z^{p}\right)
$$

Then,

$$
H_{k}(\omega)=\frac{e^{j k q \omega / p}}{p} H\left(\frac{\omega}{p}\right) \text { for } \omega \in[0, \pi] .
$$

if and only if

$$
H(\omega)=0 \quad \text { for } \omega \in[\pi / p, \pi] .
$$

Proof: See Appendix 3.

By Prop. 3, a generalization of the fractional down-sampler for arbitrary coprime pairs $p, q$ can be made as shown in Fig. 22a. Once again interpreting $e^{j k q \omega / p}$ as a fractional advance operator by $k q / p$, we see that, for an input $x(n)$, this system sets the output $y(n)=x(q n / p)$. An equivalent system to the fractional down-sampler is given in Fig $22 \mathrm{~b}$. This equivalency follows from Prop. 3. By the definition in 


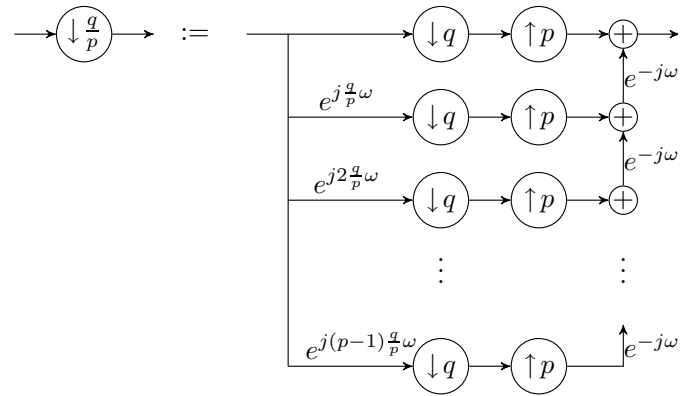

(a)

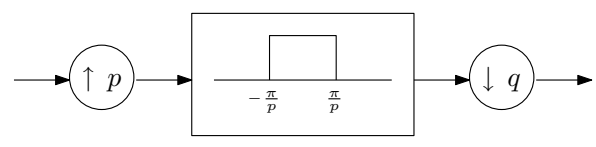

(b)

Fig. 22. Two equivalent definitions for a fractional down-sampler by $q / p$.

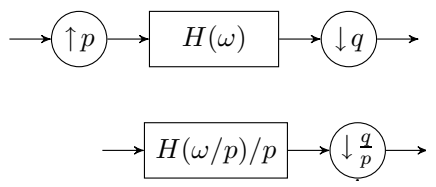

Fig. 23. The two systems are equal if and only if (55) holds.

Fig. 22a and Prop. 3, we see that the two systems in Fig. 23 are equivalent.

We can now rewrite the FB in Fig. 4. Noting that $H(\omega)$ (by (13)) satisfies (55), we define $\bar{H}(\omega)$ as in (33). Using this, we conclude that the FBs in Fig. 4 and 24 are equivalent. In Fig. 24 the filtering is applied directly to the input signal, not to the up-sampled input signal. Fig. 24 shows more explicitly the discrete-time sequences with which the input signal is convolved - namely the fractional time-shifts of $\bar{h}(n)$ and $\bar{g}(n)$.

The definition of the fractional down-sampler makes provides a clearer understanding of the spectral analysis provided by the iterated rational FBs as well. In fact, by Proposition 2 and (41) the iterated FB with $n$ stages is equivalent to the system in Fig. 25 where $\bar{H}_{n}(\omega)$ and $\bar{G}_{n}(\omega)$ are defined by (37) and (41).

\section{A. Underlying Wavelet Frame}

Using the low-pass filter $\bar{H}(\omega)$ in (33) we define the scaling function via the infinite product as

$$
\hat{\phi}(\omega)=\prod_{k=1}^{\infty} \sqrt{\frac{p}{q}} \bar{H}\left(\omega\left(\frac{p}{q}\right)^{k}\right)=\sqrt{\frac{p}{q}} \bar{H}\left(\frac{p}{q} \omega\right) \hat{\phi}\left(\frac{p}{q} \omega\right) .
$$

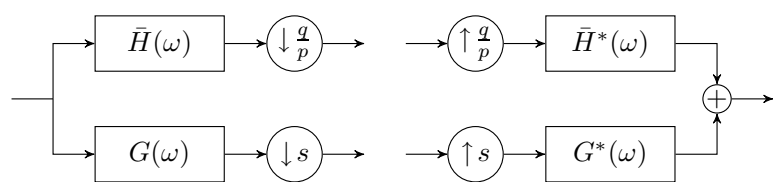

Fig. 24. This FB is equivalent to the FB in Fig. 4.

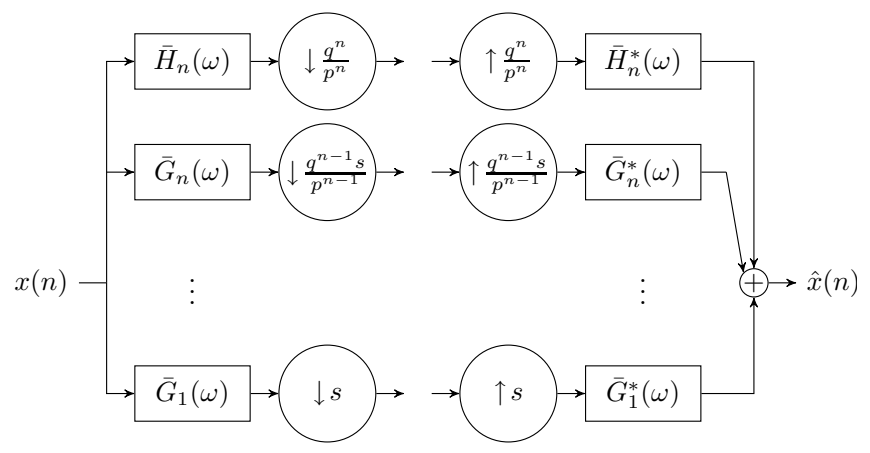

Fig. 25. The iterated FB can be interpreted as a constant-Q transform.

It follows by Prop. 2 that $\phi(t)$ is bandlimited to $[-\pi, \pi]$. The scaling function satisfies the scaling equation

$$
\phi(t)=\sqrt{\frac{q}{p}} \sum_{n} \bar{h}(n) \phi\left(\frac{q}{p} t-n\right),
$$

obtained by taking the inverse Fourier transform of (56). Based on the scaling function, we define the wavelet function as

$$
\psi(t)=\sqrt{\frac{q}{p}} \sum_{n} g(n) \phi\left(\frac{q}{p} t-n\right) .
$$

Equivalently,

$$
\begin{aligned}
\hat{\psi}(\omega) & =\sqrt{\frac{p}{q}} G\left(\frac{p}{q} \omega\right) \hat{\phi}\left(\frac{p}{q} \omega\right) \\
& =\sqrt{\frac{p}{q}} G\left(\frac{p}{q} \omega\right) \prod_{k=2}^{\infty} \sqrt{\frac{p}{q}} \bar{H}\left(\omega\left(\frac{p}{q}\right)^{k}\right) .
\end{aligned}
$$

Equivalence of this definition to the definition in (42) follows readily. We also see from (42) that $\psi(t)$ is bandlimited to $[(1-1 / s) q \pi / p, q \pi / p]$ (see Figs. 14-17). These definitions lead to,

Proposition 4: For $f(t) \in L_{2}(\mathbb{R})$, suppose we are given

$$
x(k)=\left\langle f(t), \sqrt{\frac{q}{p}} \phi\left(\frac{q}{p} t+k\right)\right\rangle .
$$

Then, (i)

1) $y_{1}(k)=\langle f(t), \phi(t+k)\rangle$ is obtained by applying the low-pass analysis system in Fig. 24 to $x(k)$.

2) $y_{2}(k)=\left\langle f(t), \psi\left(t+\frac{s p}{q} k\right)\right\rangle$ is obtained by applying the high-pass analysis system in Fig. 24 to $x(k)$.

Proof: Part (ii) follows readily from the definition of the wavelet function. For the proof of part (i), see Appendix 4. Using this result we also have

Proposition 5: The set

$$
\left\{\left(\frac{q}{p}\right)^{k / 2} \psi\left(\left(\frac{q}{p}\right)^{k} t+\frac{s p}{q} n\right)\right\}_{k, n \in \mathbb{Z}}
$$

is a tight frame for $L_{2}(\mathbb{R})$.

Proof: See Appendix E.

These propositions suggest that the rational-dilation DWT is a 'fast algorithm' for computing the coefficients of a rationaldilation wavelet frame expansion of a finite energy function (also see [2] for the critically-sampled case). 
Besides redundancy, this wavelet frame differs from Auscher's orthonormal MRA with a rational dilation factor in the number of wavelets required. With the removal of the up-sampler in the high-pass channel, the introduced wavelet frame is constructed by a single wavelet function, whereas the orthonormal MRA required $q-p$ wavelets to span $L_{2}(\mathbb{R}$ ) (also see [20] where non-refinable frame sequences are constructed, which yield an FB scheme with rational sampling factors).

\section{CONCLUSION}

This paper shows that the development of self-inverting overcomplete wavelet transforms based on rational dilations can be conveniently accomplished using the frequency-domain design of over-sampled filter banks. Even though the design and implementation is performed in the frequency domain and the discrete-time analysis/synthesis functions are not of compact support, they decay rapidly and are well localized in the time-frequency plane.

As shown in the paper, the proposed rational-dilation wavelet transform can attain a range of redundancies and Q-factors - making the transform more flexible than the dyadic wavelet transform. In particular, the proposed wavelet transform can have a substantially higher Q-factor than the dyadic wavelet transform; equivalently, it can attain improved frequency resolution. It is therefore expected that the proposed wavelet transform will provide an efficient representation of a broader class of signals: including short-time periodic/oscillatory signals like EEG, speech, and other signals arising from physical vibration phenomena. Using the proposed wavelet transform with a low Q-factor yields wavelets similar to the Mexican hat wavelet, a dense sampling of the time-frequency plane, and an exactly invertible approximation to the continuous wavelet transform. This may facilitate wavelet-based signal processing algorithms that utilize the time-scale structure of wavelet coefficients such as [10].

Additionally, in contrast to other rational-dilation discrete wavelet transforms, the proposed discrete wavelet transform corresponds to a wavelet frame for the real line based on a single wavelet, $\psi(t)$, defined through an infinite product formula.

We have used the proposed transform to decompose an EEG signal into a sum of rhythmic and transient components; one goal of this application is to improve the accuracy of the phase locking value between two EEG channels. The transform may also be useful for speech enhancement and for the restoration of clipped speech. For these applications it is typical to use the short-time Fourier transform (STFT); however, the frequency resolution of a constant-Q transform (unlike the STFT) scales with frequency. A constant-Q filter bank is a 'uniform filter bank on a log scale' which is a natural frequency spacing for analyzing and processing sounds and other oscillatory signals. For example, the human ear has a near constant-Q frequency resolution property above $500 \mathrm{~Hz}$.

\section{APPENDIX A}

\section{PROOF OF PROPOSITION 1}

Notice that $p+1=q=s$ implies that the FB is critically sampled. There exist orthonormal FIR FBs with such sampling factors. Now suppose $p+1=q=s$ is not valid. Then, we need the FB to have at least as many output samples as the input samples. This requires that, $p / q+1 / s>1$ implying $s<$ $q$. Suppose there exist filters with rational transfer functions achieving PR. To obtain a contradiction, we will provide an input that cannot be recovered. Let $D=\min (2 \pi / q, 2 \pi / s-$ $2 \pi / q)$ and define the set $B=[2 \pi / q, 2 \pi / q+D)$. Now if,

$$
X(\omega)= \begin{cases}1 & \text { for } \omega \in[0, D) \\ 0 & \text { for } \omega \in[D, 2 \pi] .\end{cases}
$$

then there exists a unique $k \in\{1,2, \ldots, q-1\}$ s.t.

$X\left(\omega+n \frac{p 2 \pi}{q}\right)=\delta_{n, k} \quad$ for $\omega \in B, \quad n=0,1, \ldots, q-1$.

Also,

$$
X\left(\omega+n \frac{2 \pi}{s}\right)=0 \quad \text { for } \omega \in B, \quad n=0,1, \ldots, s-1 .
$$

Hence we have, by (7), $Y(\omega)=L_{k}(\omega)$ for $\omega \in B$. Since $L_{k}(\omega)$ is the DTFT of a filter with a non-zero rational transfer function, it cannot be zero on a finite measure. Thus, $Y(\omega) \neq 0$ on a subset of $B$ with non-zero measure. Since $X(\omega)=0$ on $B$, the FB is not PR.

\section{APPENDIX B \\ PROOF OF PROPOSITION 2}

We begin with a lemma.

Lemma 1: Let $N, M$ be coprime and define the sets,

$$
\begin{aligned}
& \mathcal{S}_{1}=\bigcup_{k=0}^{N-1}\left[\frac{k}{N}-\frac{a}{2 N}, \frac{k}{N}+\frac{a}{2 N}\right], \\
& \mathcal{S}_{2}=\bigcup_{k=0}^{M-1}\left[\frac{k}{M}-\frac{b}{2 M}, \frac{k}{M}+\frac{b}{2 M}\right] .
\end{aligned}
$$

Also set $c=\min (a / 2 N, b / 2 M)$. Then,

$$
\left(\mathcal{S}_{1} \bigcap \mathcal{S}_{2}\right)=[-c, c]
$$

if and only if $a M+b N<2$.

Proof: $a M+b N<2$ implies that

$$
\frac{a}{2 N}+\frac{b}{2 M}<\frac{1}{M N}<\min \left(\frac{1}{M}, \frac{1}{N}\right) .
$$

This ensures that $[-a / 2 N, a / 2 N]$ and $[-b / 2 M, b / 2 M]$ do not intersect with $[1 / M-b / 2 M, 1 / M+b / 2 M]$ and $[1 / N-$ $a / 2 N, 1 / N+a / 2 N]$ respectively.

For the remaining part, we will prove the contrapositive of the statement. Suppose (67) is not true. This is equivalent to saying that there exist integers $n, k$ with $1 \leq n \leq M-1$, $1 \leq k \leq N-1$ s.t.

$$
\frac{k}{N}-\frac{a}{2 N}-\frac{b}{2 M} \leq \frac{n}{M} \leq \frac{k}{N}+\frac{a}{2 N}+\frac{b}{2 M},
$$

or,

$$
\frac{M k}{N}-\frac{a M}{2 N}-\frac{b}{2} \leq n \leq \frac{M k}{N}+\frac{a M}{2 N}+\frac{b}{2} .
$$

For $M, N$ coprime, since $\{(M k \bmod N)\}_{k=1}^{N-1}=$ $\{1,2, \ldots, N\}$, we see that (70) is true if and only if, 


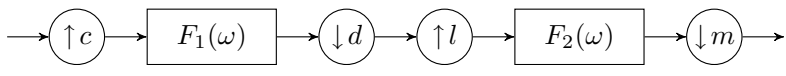

Fig. 26. Two rate changers in cascade, used in Corollary 1.

$a M / 2 N+b / 2 \geq 1 / N$, which is equivalent to $a M+b N \geq 2$

As a corollary, we have,

Corollary 1: Suppose that $F_{1}(\omega)$ and $F_{2}(\omega)$ are filters bandlimited to $\pi / d$ and $b \pi$ (with $b<1 / l$ ), respectively. Suppose also that $l<m$. Then, $F_{1}(l \omega) F_{2}(d \omega)$ is bandlimited to $b \pi / d$.

Proof: Notice that the frequency support of $F_{1}(l \omega)$ is a subset of

$$
\mathcal{S}_{1}=\bigcup_{k=0}^{l-1}\left[\frac{2 \pi k}{l}-\frac{\pi}{d l}, \frac{2 \pi k}{l}+\frac{\pi}{d l}\right]
$$

Similarly, the frequency support of $F_{2}(d \omega)$ is a subset of

$$
\mathcal{S}_{2}=\bigcup_{k=0}^{d-1}\left[\frac{2 \pi k}{d}-\frac{b \pi}{d}, \frac{2 \pi k}{d}+\frac{b \pi}{d}\right] .
$$

For $a=1 / d, N=l$ and $M=d$, we also have $a M+b N<2$. The corollary now follows by Lemma 1.

Using this corollary, Prop. 2 follows by an induction argument.

\section{APPENDIX C \\ ProOF OF PROPOSITION 3}

In this appendix we will deviate from the convention adopted in the rest of the paper and take the domain of the DTFT to be $[-\pi, \pi]$. We remark that the domain was $[0, \pi]$ in the statement of the Prop. 3.

Proof: Notice that $H_{k}(\omega)$ can be obtained from $H(\omega)$ via,

$$
\begin{array}{r}
H_{k}(p \omega)=\frac{1}{p} \sum_{n=0}^{p-1} A_{k}\left(\omega+n \frac{2 \pi}{p}\right) H\left(\omega+n \frac{2 \pi}{p}\right) \\
\text { for } \omega \in\left[-\frac{\pi}{p}, \frac{\pi}{p}\right],
\end{array}
$$

where $A_{k}(\omega)$ is the $2 \pi$-periodic function equal to $e^{j k q \omega / p}$ on $\omega \in[-\pi, \pi]$. Now notice that,

$$
A_{k}\left(\omega+n \frac{2 \pi}{p}\right)=e^{j k q \omega} e^{j n k q 2 \pi / p} \text { for } \omega \in\left[-\frac{\pi}{p}, \frac{\pi}{p}\right] .
$$

Inserting this in (73), we get

$$
\begin{array}{r}
H_{k}(p \omega)=\frac{e^{j k q \omega}}{p} H(\omega)+\frac{e^{j k q \omega}}{p} \sum_{n=1}^{p-1} e^{j n k q 2 \pi / p} H\left(\omega+n \frac{2 \pi}{p}\right) \\
\text { for } \omega \in\left[-\frac{\pi}{p}, \frac{\pi}{p}\right] . \quad
\end{array}
$$

Thus (54) holds if and only if

$$
\begin{aligned}
& \sum_{n=1}^{p-1} e^{j n k q 2 \pi / p} H\left(\omega+n \frac{2 \pi}{p}\right)=0 \\
& \qquad \text { for } \omega \in\left[-\frac{\pi}{p}, \frac{\pi}{p}\right] \text { and } k=1,2, \ldots, p-1,
\end{aligned}
$$

or equivalently,

$$
\mathbf{W}\left(\begin{array}{c}
H\left(\omega+\frac{2 \pi}{p}\right) \\
H\left(\omega+2 \frac{2 \pi}{p}\right) \\
\vdots \\
H\left(\omega+(p-1) \frac{2 \pi}{p}\right)
\end{array}\right)=\mathbf{0} \text { for } \omega \in\left[-\frac{\pi}{p}, \frac{\pi}{p}\right]
$$

where $\mathbf{W}$ is a $(p-1) \times(p-1)$ matrix whose $(k, n)$ th entry is $e^{j n k q 2 \pi / p}$. Noting that $\mathbf{W}$ is an orthonormal matrix, we see that (77) is equivalent to

$$
\begin{aligned}
& H\left(\omega+n \frac{2 \pi}{p}\right)=0 \\
& \qquad \text { for } \omega \in\left[-\frac{\pi}{p}, \frac{\pi}{p}\right], \quad n=1,2, \ldots, p-1,
\end{aligned}
$$

which is equivalent to $(55)$.

\section{APPENDIX D \\ ProOF OF PROPOSITION 4}

First let us note that,

$$
\mathcal{F}\left\{\sqrt{\frac{q}{p}} \phi\left(\frac{q}{p} t+k\right)\right\}=\sqrt{\frac{p}{q}} e^{j \omega k p / q} \Phi\left(\frac{p \omega}{q}\right),
$$

where $\mathcal{F}$ denotes the Fourier transform operator. Using the Plancherel formula, we can write $x(k)$ as,

$$
\begin{aligned}
x(k) & =\frac{1}{2 \pi} \int_{-\infty}^{\infty} \hat{f}(\omega) \sqrt{\frac{p}{q}} e^{-j \omega k p / q} \Phi^{*}\left(\frac{p \omega}{q}\right) d \omega \\
& =\frac{1}{2 \pi} \int_{-\infty}^{\infty} \hat{f}\left(\frac{q \omega^{\prime}}{p}\right) \sqrt{\frac{q}{p}} \Phi^{*}\left(\omega^{\prime}\right) e^{-j \omega^{\prime} k} d \omega^{\prime} \\
& =\frac{1}{2 \pi} \int_{-\pi}^{\pi}\left\{\sqrt{\frac{q}{p}} \hat{f}\left(-\frac{q \omega}{p}\right) \Phi^{*}(-\omega)\right\} e^{j \omega k} d \omega
\end{aligned}
$$

In the last line we used the fact that $\phi(t)$ is bandlimited to $[-\pi, \pi]$. We recognize the term in the brackets as the DTFT of $x(k)$, that is $X(\omega)$. Now,

$$
\begin{gathered}
\langle f(t), \phi(t+p k+m)\rangle \\
=\frac{1}{2 \pi} \int_{-\infty}^{\infty} \hat{f}(\omega) \sqrt{\frac{p}{q}} \bar{H}^{*}\left(\frac{p}{q} \omega\right) \hat{\phi}^{*}\left(\frac{p \omega}{q}\right) e^{-j(p k+m) \omega} d \omega \\
=\frac{1}{2 \pi} \int_{-\pi}^{\pi} \hat{f}\left(-\frac{q \omega}{q}\right) \sqrt{\frac{q}{p}} \Phi^{*}(-\omega) \\
\quad \cdot \bar{H}^{*}(-\omega) e^{j q m \omega / p} e^{j q k \omega} d \omega \\
=\frac{1}{2 \pi} \int_{-\pi}^{\pi}\left\{X(\omega) H(\omega) e^{j q m \omega / p}\right\} e^{j q k \omega} .
\end{gathered}
$$

where we used $\bar{H}^{*}(-\omega)=\bar{H}(\omega)$ in the last line. This means that

$$
y_{1}(p k+m)=(\downarrow q) T^{q m / p}\{x(k) * h(k)\} .
$$

where $(\downarrow q)$ denotes the down-sampling operator by $q$ and $T^{q m / p}$ denotes the fractional shift operator by $q m / p$. Notice that one obtains the low-pass system in Fig. 24 by interlacing

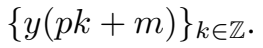




\section{APPENDIX E}

\section{PROOF OF PROPOSITION 5}

The proof is an adaptation of the proof of the unitary extension principle given by Benedetto and Treiber in [4]. We will invoke the following lemma from [13].

Lemma 2 (Lemma 5.1.7 in [13]): Suppose that $\left\{f_{k}\right\}_{k \in \mathbb{Z}}$ is a sequence of elements in $\mathcal{H}$ and that there exist constants $A, B>0$ s.t.

$$
A\|f\|^{2} \leq \sum_{k \in \mathbb{Z}}\left|\left\langle f, f_{k}\right\rangle\right|^{2} \leq B\|f\|^{2}
$$

for all $f$ in a dense subset $V$ of $\mathcal{H}$. Then $\left\{f_{k}\right\}_{k \in \mathbb{Z}}$ is a frame for $\mathcal{H}$ with bounds $A, B$.

Proof of Prop. 5: First notice that

$$
\hat{\phi}(\omega)=1 \quad \text { for } \omega \in\left[-\frac{q}{p}\left(1-\frac{1}{s}\right) \pi, \frac{q}{p}\left(1-\frac{1}{s}\right) \pi\right] .
$$

Noting also that $\hat{\phi}(\omega)=0$ for $|\omega|>\pi$, we have

$$
\begin{aligned}
& \sum_{n=-\infty}^{\infty}|\hat{\phi}(\omega+n 2 \pi)|^{2}=1 \\
& \quad \text { for } \omega \in\left[-\frac{q}{p}\left(1-\frac{1}{s}\right) \pi, \frac{q}{p}\left(1-\frac{1}{s}\right) \pi\right] .
\end{aligned}
$$

This implies (see [13], Chp. 7) that $\{\phi(t-n)\}_{n \in \mathbb{Z}}$ is a tight frame sequence for functions bandlimited to $q / p(1-1 / s) \pi$. Likewise we have that $\left\{(q / p)^{k / 2} \phi\left((q / p)^{k} t-n\right)\right\}_{n \in \mathbb{Z}}$ is a tight frame sequence for functions bandlimited to $(q / p)^{k+1}(1-1 / s) \pi$.

Now take a bandlimited function $f(t)$ with unit norm, otherwise arbitrary. For this function, define the sequences $c_{k}(n), d_{k}(n)$ as,

$$
\begin{aligned}
& c_{k}(n)=\left\langle f(t),(q / p)^{k / 2} \phi\left((q / p)^{k} t+n\right)\right\rangle, \\
& d_{k}(n)=\left\langle f(t),(q / p)^{k / 2} \psi\left((q / p)^{k} t+(s p / q) n\right)\right\rangle .
\end{aligned}
$$

By the fact previously stated, we can find an integer $K$ s.t. for integer $r \geq 0$,

$$
\sum_{n \in \mathbb{Z}}\left|c_{K+r}(n)\right|^{2}=1
$$

Since $\hat{\psi}(\omega)$ is bandlimited to $[(1-1 / s) q \pi, q \pi / p]$, we also have that

$$
d_{K+r}(n)=0 \quad \text { for } r \geq 0 .
$$

Now let us take a look at the other end of the integer lattice. Pick an $\epsilon>0$. For this $\epsilon$, we can find integer $N$ s.t. for integer $l \geq 0$,

$$
\sum_{n \in \mathbb{Z}}\left|c_{N-l}(n)\right|^{2}<\epsilon .
$$

Now, given $c_{N}$, by Prop. 4, we can obtain $c_{N-l}, d_{N-l}, d_{N-l+1}, \ldots, d_{N-1}$ by applying the $l$-stage FB to $c_{N}$. Since this FB is also a tight frame for $l_{2}(\mathbb{Z})$, we have

$$
\sum_{n \in \mathbb{Z}}\left|c_{N-l}(n)\right|^{2}+\sum_{m=1}^{l} \sum_{n \in \mathbb{Z}}\left|d_{N-m}(n)\right|^{2}=\sum_{n \in \mathbb{Z}}\left|c_{N}(n)\right|^{2} .
$$

Thus we have, for arbitrary $l, \sum_{m=1}^{l} \sum_{n \in \mathbb{Z}}\left|d_{N-m}(n)\right|^{2}<\epsilon$, implying

$$
\sum_{k=-\infty}^{N-1} \sum_{n \in \mathbb{Z}}\left|d_{k}(n)\right|^{2}<\epsilon
$$

Now let us look at $d_{k}(n)$ for $N<k<K$. Again applying a $K-N$ stage $\mathrm{FB}$, and noting that this $\mathrm{FB}$ is a tight frame for $l_{2}(\mathbb{Z})$, we get

$1>\sum_{k=N}^{K-1} \sum_{n \in \mathbb{Z}}\left|d_{k}(n)\right|^{2}=\sum_{n \in \mathbb{Z}}\left|c_{K}(n)\right|^{2}-\sum_{n \in \mathbb{Z}}\left|c_{N}(n)\right|^{2}>1-\epsilon$.

By (91), (94) then

$$
1+\epsilon>\sum_{k=-\infty}^{\infty} \sum_{n \in \mathbb{Z}}\left|d_{k}(n)\right|^{2}>1-\epsilon .
$$

By the arbitrariness of $\epsilon$, we conclude that $\left\{(q / p)^{k / 2} \psi\left((q / p)^{k} t+(s p / q) n\right)\right\}_{k, n \in \mathbb{Z}}$ is a tight frame for bandlimited functions. Since bandlimited functions are dense in $L_{2}(\mathbb{R})$, the statement follows by Lemma 2 .

\section{REFERENCES}

[1] P. Auscher. Wavelet bases for $L^{2}(R)$ with rational dilation factor. In M. B. Ruskai et al., editor, Wavelets and Their Applications. Jones and Barlett, Boston, 1992.

[2] A. Baussard, F. Nicolier, and F. Truchetet. Rational multiresolution analysis and fast wavelet transform: application to wavelet shrinkage denoising. Signal Processing, 84(10):1735-1747, October 2004.

[3] İ. Bayram and I. W. Selesnick. Overcomplete discrete wavelet transforms with rational dilation factors. IEEE Trans. Signal Processing, 57(1):131145, January 2009.

[4] J. J. Benedetto and O. Treiber. Wavelet frames: Multiresolution analysis and extension principles. In L. Debnath, editor, Wavelet Transforms and Time-Frequency Signal Analysis. Birkhäuser, 2001.

[5] P. Blanc, T. Blu, T. Ranchin, L. Wald, and R. Aloisi. Using iterated filter banks within the ARSIS concept for producing $10 \mathrm{~m}$ Landsat multispectral images. International Journal of Remote Sensing, 41(12):2331 - 2343, August 1998.

[6] T. Blu. Iterated filter banks with rational rate changes - connection with discrete wavelet transforms. IEEE Trans. Signal Processing, 41(12):3232-3244, December 1993.

[7] T. Blu. An iterated rational filter bank for audio coding. In Proc. IEEE Int. Symposium on Time-frequency and Time-scale Analysis, 1996.

[8] J. C. Brown. Calculation of a constant Q spectral transform. J. Acoust. Soc. Amer., 89(1):425-434, January 1991.

[9] J. C. Brown and M. S. Puckette. An efficient algorithm for the calculation of a constant Q transform. J. Acoust. Soc. Amer., 92(5):26982701, November 1992

[10] V. Bruni, B. Piccoli, and D. Vitulano. A fast computation method for time scale signal denoising. Signal, Image and Video Processing, 3(1):63-83, February 2009.

[11] C. S. Burrus, R. Gopinath, and H. Guo. Introduction to Wavelets and Wavelet Transforms: A Primer. Prentice Hall, 1997.

[12] G. F. Choueiter and J. R. Glass. An implementation of rational wavelets and filter design for phonetic classification. IEEE Trans. Audio, Speech and Language Processing, 15(3):939-948, March 2007.

[13] O. Christensen. An Introduction to Frames and Riesz Bases. Birkhäuser, 2003.

[14] C. Chui and W. He. Compactly supported tight frames associated with refinable functions. J. of Appl. and Comp. Harm. Analysis, 8(3):293319, May 2000.

[15] L. Cohen. Time-Frequency Analysis. Prentice Hall, 1995.

[16] I. Daubechies. Ten Lectures on Wavelets. SIAM, 1992.

[17] F. C. C. B. Diniz, I. Koethe, S. L. Netto, and L. W. P. Biscainho. Highselectivity filter banks for spectral analysis of music signals. EURASIP Journal on Advances in Signal Processing, 2007:Article ID 94704, 12 pages, 2007. doi:10.1155/2007/94704. 
[18] M. Feilner, D. Van De Ville, and M. Unser. An orthogonal family of quincunx wavelets with continuously adjustable order. IEEE Trans. Image Processing, 14(4):499-510, April 2005.

[19] M. Holschneider, R. Kronland-Martinet, J. Morlet, and A. Grossmann. A real-time algorithm for signal analysis with the help of the wavelet transform. In J. M. Combes, A. Grossmann, and Ph. Tchamitchian, editors, Wavelets, Time-Frequency Methods and Phase Space, pages 286-297. Springer-Verlag, 1989.

[20] B. D. Johnson. Stable filtering schemes with rational dilations. J. of Fourier Analysis and App., 13(5):607-621, 2007.

[21] A. Karmakar, A. Kumar, and R. K. Patney. Design of optimal wavelet packet trees based on auditory perception criterion. IEEE Signal Processing Letters, 14(4):240-243, April 2007.

[22] J. Kovačević and A. Chebira. Life beyond bases: The advent of frames (Part I). IEEE Signal Processing Magazine, 24(4):86-104, July 2007.

[23] J. Kovačević and M. Vetterli. Perfect reconstruction filter banks with rational sampling factors. IEEE Trans. Signal Processing, 41(6):20472066, June 1993.

[24] J. Lin and M. J. T. Smith. New perspectives and improvements on the symmetric extension filter bank for subband/wavelet image compression. IEEE Trans. Image Processing, 17(17):177-180, February 2008.

[25] A. Makur and S. K. Mitra. Warped discrete-fourier transform: Theory and applications. IEEE Trans. Circuits and Systems-I:Fund. Theory and App., 48(9):1086-1093, September 2001.

[26] S. K. Mitra. Digital Signal Processing. McGraw-Hill, $3^{\text {rd }}$ edition, 2005.

[27] B. Mont-Reynaud. The bounded-Q approach to time-varying spectral analysis. Stanford University, Department of Music Technical Report, STAN-M-28, 1986.

[28] A. V. Oppenheim, D. H. Johnson, and K. Steiglitz. Computation of spectra with unequal resolution using the fast Fourier transform. Proc. IEEE, 59(2):299-301, February 1971.

[29] I. W. Selesnick. The double density DWT. In A. Petrosian and F. G. Meyer, editors, Wavelets in Signal and Image Analysis: From Theory to Practice. Kluwer, 2001.

[30] I. W. Selesnick, R. G. Baraniuk, and N. G. Kingsbury. The dual-tree complex wavelet transform - A coherent framework for multiscale signal and image processing. IEEE Signal Processing Magazine, 22(6):123151, November 2005.

[31] E. P. Simoncelli, W. T. Freeman, E. H. Adelson, and D. J. Heeger. Shiftable multi-scale transforms. IEEE Trans. Information Theory, 38(2):587-607, March 1992.

[32] J. O. Smith and J. S. Abel. Bark and ERB bilinear transforms. IEEE Trans. Speech and Audio Processing, 7(6):697-708, November 1999.

[33] P.P. Vaidyanathan. Multirate Systems and Filter Banks. Prentice Hall, 1992.

[34] D. Van De Ville, T. Blu, and M. Unser. Isotropic polyharmonic Bsplines: Scaling functions and wavelets. IEEE Trans. Image Processing, 14(11):1798-1813, November 2005. 